\title{
ANOSOV DIFFEOMORPHISMS AND EXPANDING IMMERSIONS. II
}

\author{
BY
}

LOWELL JONES

\begin{abstract}
This paper continues the study of hyperbolic attractors, expanding immersions, and quotient solenoids which was begun in a previous paper of the same title. The main result states that certain hyperbolic attractors are topologically conjugate to an Anosov diffeomorphism.
\end{abstract}

0. Introduction. This paper continues the study of shift maps on quotient solenoids begun in $[\mathbf{4}]$.

Let $f: K \rightarrow K$ denote an expanding immersion on a compact branched manifold without boundary, which is equipped with an L.C.S. $[\mathbf{4}, \S 1]$. Let $F^{\prime}: \Sigma^{\prime}(f) \rightarrow \Sigma^{\prime}(f)$ denote the associated shift map on the quotient solenoid $[4, \S 2]$. The main result of this paper is the following.

THEOREM 0.1. If $\operatorname{dim}(K)=1$, then $F^{\prime}: \Sigma^{\prime}(f) \rightarrow \Sigma^{\prime}(f)$ is topologically conjugate to a codimension one Anosov diffeomorphism. In particular, if $\Sigma^{\prime}(f)$ is connected, then $F^{\prime}: \Sigma^{\prime}(f) \rightarrow \Sigma^{\prime}(f)$ is conjugate to a linear Anosov diffeomorphism on a torus.

Because of the relationship which exists between shift maps on quotient solenoids and hyperbolic attractors $[\mathbf{4}, 2.7]$, there is the following corollary of 0.1 .

COROLLARY 0.2. Let $g: M \rightarrow M$ denote a diffeomorphism of the smooth manifold $M$, and $\Lambda \subset M^{0}$ a compact hyperbolic attractor for $g$ having a $C^{1}$, codimension one stable foliation such that $\Lambda \cap W^{s}(x)$ is path connected for every stable leaf $W^{s}(x), x \in \Lambda$. Then $g: \Lambda \rightarrow \Lambda$ is topologically conjugate to a codimension one Anosov diffeomorphism.

Note 0.1 verifies a special case of Conjecture 2.5 in [4], whereas 0.2 verifies a special case of S. Smale's attractor conjecture (see $[\mathbf{4}, \S 2]$ ).

Theorem 0.1 is proven in $\S 3$. In $\S 1$ local properties of shift maps on quotient solenoids are studied with no restrictions on $\operatorname{dim}(K)$. In $\S 2$ a proposition $(2.1)$ is proven which is needed in the proof of Theorem 0.1 .

Of the many sources the author has used in the preparation of this paper, he has been especially guided by the work of J. Franks $[\mathbf{2}]$, A. Manning $[\mathbf{5}, \mathbf{6}]$, and S. E. Newhouse [7].

1. Local properties of quotient solenoids. Before stating the main result of this section, it will be useful to make some definitions. A local product structure on a topological space $S$ consists of an open covering $\left\{W_{i}\right\}$ for $S$ and homeomorphisms

Received by the editors February 19, 1985 .

1980 Mathematics Subject Classification. Primary 58F15, 57R42. 
$\left\{h_{i}: W_{i} \rightarrow X_{i} \times Y_{i}\right\}$ onto cartesian product spaces, satisfying:

1.1. (a) For each pair $i, j, h_{i}\left(W_{i} \cap W_{j}\right)=X_{i j} \times Y_{i j} \subset X_{i} \times Y_{i}$.

(b) Each composition

$$
X_{j i} \times Y_{j i} \stackrel{h_{j}^{-1}}{\rightarrow} W_{i} \cap W_{j} \stackrel{h_{i}}{\rightarrow} X_{i j} \times Y_{i j}
$$

is a cartesian product of homeomorphisms $r_{j i}: X_{j i} \rightarrow X_{i j}$ and $s_{j i}: Y_{j i} \rightarrow Y_{i j}$.

A homeomorphism $g: S \rightarrow S$ preserves the local product structure 1.1(a), (b) if for any $i, j$ and $x$ with $x \in W_{i}, g(x) \in W_{j}$, there are open subsets $X_{i}^{\prime} \subset X_{i}, Y_{i}^{\prime} \subset$ $Y_{i}, X_{j}^{\prime} \subset X_{j}, Y_{j}^{\prime} \subset Y_{j}$ such that $x \in h_{i}^{-1}\left(X_{i}^{\prime} \times Y_{i}^{\prime}\right), g(x) \in h_{j}^{-1}\left(X_{j}^{\prime} \times Y_{j}^{\prime}\right)$, and each composition

$$
X_{i}^{\prime} \times Y_{i}^{\prime} \stackrel{h_{i}^{-1}}{\rightarrow} W_{i} \stackrel{g}{\rightarrow} N \supset h_{j}^{-1}\left(X_{j}^{\prime} \times Y_{j}^{\prime}\right) \stackrel{h_{j}}{\rightarrow} X_{j}^{\prime} \times Y_{j}^{\prime}
$$

is a cartesian product of homeomorphisms $X_{i}^{\prime} \cong X_{j}^{\prime}, Y_{i}^{\prime} \cong Y_{j}^{\prime}$.

Proposition 1.2. Let $F^{\prime}: \Sigma^{\prime}(f) \rightarrow \Sigma^{\prime}(f)$ denote the shift map on the quotient solenoid of an arbitrary $C^{r}$ expanding immersion $f: K \rightarrow K$ equipped with a W.L.C.S. The following are true.

(a) $\Sigma^{\prime}(f)$ is a compact metric space, and $F^{\prime}$ is a homeomorphism.

(b) $\Sigma^{\prime}(f)$ has a local product structure as in 1.1 , which $F^{\prime}$ preserves.

(c) $F^{\prime}: \Sigma^{\prime}(f) \rightarrow \Sigma^{\prime}(f)$ is contracting in the $X_{i}$ factors. That is, there is $\delta>$ $0, a>0,1>\lambda>0$ so that for any $i, p \in Y_{i}$ if $y_{1}, y_{2} \in X_{i} \times p$ and $d\left(y_{1}, y_{2}\right)<\delta$, then $d\left(F^{\prime n}\left(y_{1}\right), F^{\prime n}\left(y_{2}\right)\right)<a \lambda^{n} d\left(y_{1}, y_{2}\right)$ for all integers $n>0$. Here $d($,$) is the$ metric of $(a)$ above.

(d) $F^{\prime-1}: \Sigma^{\prime}(f) \rightarrow \Sigma^{\prime}(f)$ is contracting in the $Y_{i}$ factors.

(e) Define the stable and unstable leaves of $F^{\prime}: \Sigma^{\prime}(f) \rightarrow \Sigma^{\prime}(f)$, through the point $x \in \Sigma^{\prime}(f)$, by

$$
\begin{gathered}
W^{s}(x) \equiv\left\{y \in \Sigma^{\prime}(f): \lim _{n \rightarrow \infty} d\left(f^{n}(x), f^{n}(y)\right)=0\right\}, \\
W^{u}(x) \equiv\left\{y \in \Sigma^{\prime}(f): \lim _{n \rightarrow \infty} d\left(f^{-n}(x), f^{-n}(y)\right)=0\right\} .
\end{gathered}
$$

Then each $W^{u}(x)$ is a one-to-one continuous image of $R^{k}(k=\operatorname{dim}(K))$. If the W.L.C.S. for $f: K \rightarrow K$ is actually an L.C.S., then each $W^{s}(x)$ is path connected.

(f) $\Sigma^{\prime}(f)$ has finite topological dimension.

REMARK 1.3. Note if Conjecture 2.6 of $[4]$ could be verified, then Smale's attractor conjecture (see $[4, \S 2]$ ) would imply the following weak form of Conjecture 2.5 of [4]: every shift map $F^{\prime}: \Sigma^{\prime}(f) \rightarrow \Sigma^{\prime}(f)$ associated to an L.C.S. is an Anosov homeomorphism (a homeomorphism of a compact manifold without boundary, having local expanding and local contracting direction in the sense of 1.2(c), (d)). To see this, note by $[\mathbf{4}, 2.6]$ and $1.2(\mathrm{e})$ that $F^{\prime}: \Sigma^{\prime}(f) \rightarrow \Sigma^{\prime}(f)$ would be a hyperbolic attractor with each $\Sigma^{\prime}(f) \cap W^{s}(x)$ path connected. The only such attractors which satisfy the attractor conjecture must be manifolds.

PROOF OF PROPOSITION 1.2. First $1.2(\mathrm{a})$ will be verified. $\Sigma(f)$ is a compact space (see [10]); so the quotient space $\Sigma^{\prime}(f) \equiv \Sigma(f) / \sim$ is also compact. The shift map $F: \Sigma(f) \rightarrow \Sigma(f)$ on the solenoid $\Sigma(f)$ is a homeomorphism [11]. Its inverse $F^{-1}: \Sigma(f) \rightarrow \Sigma(f)$ also preserves the equivalence relation $\sim$ on $\Sigma(f)$, so its quotient $\left(F^{-1}\right)^{\prime}: \Sigma^{\prime}(f) \rightarrow \Sigma^{\prime}(f)$ is well defined. Note that $F^{\prime} \circ\left(F^{-1}\right)^{\prime}=\left(F^{-1}\right)^{\prime} \circ F^{\prime}=1$, so 
$F^{\prime}$ is a homeomorphism. A metric for $\Sigma^{\prime}(f)$ will be constructed later in this proof when $1.2(\mathrm{c}),(\mathrm{d})$ are verified.

Before proceeding with the rest of the proof of 1.2 , we state the following lemma.

LEMMA 1.4. There is for each $U_{i}$ of $[\mathbf{4}, 1.1]$ a compact subset $U_{i}^{\prime} \subset U_{i}^{0}$ satisfying the following.

(a) $\left\{\left(U_{i}^{\prime}\right)^{0}\right\}$ is an open cover of $K$.

(b) For each $x \in K$ there is a $U_{i}^{\prime}$ so that $f^{q}\left(K_{x}\right) \subset\left(U_{i}^{\prime}\right)^{0}$ and $\pi_{i}\left(f^{q}\left(K_{x}\right)\right)$ is a single point (here $q, K_{x}$ come from $[\mathbf{4}, 1.2(\mathrm{~b})]$, and $\pi_{i}$ comes from $[4,1.1]$ ).

The proof of 1.4 uses $[4,1.2(b)]$ and the compactness of $K$. The details are left to the reader.

Now to verify $1.2(\mathrm{~b})$ choose for any $x \in \Sigma^{\prime}(f)$ a representative $\bar{x}=\left(x_{0}, x_{1}, x_{2}, \ldots\right)$ for $x$ in $\Sigma(f)$. It follows from 1.4 that there is a sequence $U_{\phi(0)}, U_{\phi(1)}, U_{\phi(2)}, \ldots$ of the $\left\{U_{i}\right\}$ of $[4,1.1]$ so that if $\bar{y}=\left(y_{0}, y_{1}, y_{2}, \ldots\right)$ is any point of $\Sigma(f)$ with $x_{i}, y_{i} \in U_{i^{\prime}}, \pi_{i^{\prime}}\left(x_{i}\right)=\pi_{i^{\prime}}\left(y_{i}\right)$ for some $i^{\prime}$ and all $i \leq r$, then $x_{j}, y_{j} \in\left(U_{\phi(j)}^{\prime}\right)^{0}$ with $\pi_{\phi(j)}\left(x_{j}\right)=\pi_{\phi(j)}\left(y_{j}\right)$ for all $j \leq r-q$. For each integer $l>0$ define a subset $W_{x, l} \subset \Sigma^{\prime}(f)$ as follows: $y \in W_{x, l}$ if and only if $y$ has a representative $\bar{y}=$ $\left(y_{0}, y_{1}, y_{2}, \ldots\right)$ in $\Sigma(f)$, so that for all $i \leq l$ there is a smooth path $g_{i}:[0,1] \rightarrow U_{\phi(i)}^{0}$ such that $\pi_{\phi(i)}\left(x_{i}\right)=\pi_{\phi(i)}\left(g_{i}(0)\right), g_{i}(1)=y_{i}$ and the length of each composite path $\pi_{\phi(0)} \circ f^{i} \circ g_{i}$ is less than or equal to $2^{-l}$ with respect to the Euclidean distance on $X_{\phi(0)}=$ image $\left(\pi_{\phi(0)}\right)$. Let $Y_{x, l}$ denote the ball of radius $2^{-l}$ in $X_{\phi(0)}$ centered at $\pi_{\phi(0)}\left(x_{0}\right)$; define $r: W_{x, l} \rightarrow Y_{x, l}$ by $r(y)=\pi_{\phi(0)}\left(y_{0}\right)$, where $\bar{y}=\left(y_{0}, y_{1}, y_{2}, \ldots\right)$ is a representative for $y$ as above. Set $X_{x, l} \equiv\left\{y \in W_{x, l} \mid r(y)=r(x)\right\}$. For any $y \in$ $W_{x, l}$ with representative $\left(y_{0}, y_{1}, y_{2}, \ldots\right)=\bar{y}$ as above, there is $y^{\prime} \in X_{x, l-q}$ having representative $\left(y_{0}^{\prime}, y_{1}^{\prime}, y_{2}^{\prime}, \ldots\right)=\bar{y}^{\prime}$ in $\Sigma(f)$, so that for each $i$ we have $y_{i}, y_{i}^{\prime} \in B_{i}$, where $B_{i}$ is a smooth ball in $K$ such that $\pi_{\phi(0)} \circ f^{i}: B_{i} \rightarrow Y_{l, x}$ is a homeomorphism. Define $s: W_{x, l} \rightarrow X_{x, l-q}$ by $s(y) \equiv y^{\prime}$. It is seen (using $\left.[4,1.1,1.2(\mathrm{~b})], 1.4\right)$ that $s \times r: W_{x, l} \rightarrow X_{x, l-q} \times Y_{x, l}$ is a well-defined embedding onto a neighborhood of $(s(x), r(x))$ for sufficiently large $l$, and $\left\{W_{l, x}\right\}$ is a neighborhood system of $x$ in $\Sigma^{\prime}(f)$. Choose subsets $X_{x, l-q}^{\prime} \subset X_{x, l-q}$ and $Y_{x, l}^{\prime} \subset Y_{x, l}$ so that $X_{x, l-q} \times Y_{x, l} \subset$ image $(s \times r)$ and $W_{x, l}^{\prime} \equiv(s \times r)^{-1}\left(X_{x, l-q}^{\prime} \times Y_{x, l}^{\prime}\right)$ is an open neighborhood for $x$ in $\Sigma^{\prime}(f)$. For the $\left\{W_{i}\right\}$ of 1.1 choose a finite covering of $\Sigma^{\prime}(f)$ by the sets $\left\{W_{x, l}^{\prime}\right\}$; and set $Y_{i}=Y_{x, l}^{\prime}, X_{i}=X_{x, l-q}^{\prime}, h_{i}=s \times\left. r\right|_{W_{x, l}^{\prime}}$ for the pair $(x, l)$ corresponding to $i$. Verifying that this gives a local product structure to $\Sigma^{\prime}(f)$, which is preserved by $F^{\prime}: \Sigma^{\prime}(f) \rightarrow \Sigma^{\prime}(f)$, is a straightforward computation and is left to the reader.

Before proving 1.2(c), (d), the metric $d: \Sigma^{\prime}(f) \times \Sigma^{\prime}(f) \rightarrow R$ must be constructed. Towards this end define a real valued (noncontinuous) function $D: \Sigma^{\prime}(f) \times \Sigma^{\prime}(f) \rightarrow$ $R$ by

$$
D(x, y) \equiv \beta^{-l},
$$

where $l$ is the greatest integer such that $y \in W_{x, l}$, and $\beta$ is some number satisfying $\beta>1$ (we use the convention that $W_{x, 0}=\Sigma^{\prime}(f)$ ).

We shall need the following lemma to complete the construction of $d($,$) and$ the verification of $1.2(\mathrm{c}),(\mathrm{d})$. It will be proven at the end of this section.

LEMMA 1.6. There is $\varepsilon>0$ so that if $\beta$ in (1.5) is chosen so $1<\beta<1+\varepsilon$, then $D($,$) will satisfy the following properties.$ 
(a) For each $p \in \Sigma^{\prime}(f), n \in Z^{+}$, the sets $U_{p, n} \equiv\left\{p^{\prime} \in \Sigma^{\prime}(f): D\left(p, p^{\prime}\right) \leq 2^{-n}\right\}$ constitute a neighborhood system for $p$ in $\Sigma^{\prime}(f)$.

(b) $D\left(p, p^{\prime}\right)=0 \Leftrightarrow p=p^{\prime}$.

(c) There is a number $\gamma>0$ so that for any finite sequence $p_{1}, p_{2}, \ldots, p_{n} \in \Sigma^{\prime}(f)$ we have

$$
D_{\left(p_{1}, p_{n}\right)}<\gamma\left(\sum_{i=1}^{n-1} D\left(p_{i}, p_{i+1}\right)\right) .
$$

(d) $F^{\prime}: \Sigma^{\prime}(f) \rightarrow \Sigma^{\prime}(f)$ satisfies $1.2(\mathrm{c})$, (d) when $D($, ) replaces $d($, ).

Now define

$$
d\left(p, p^{\prime}\right) \equiv \min \left[\sum_{i=1}^{n-1}\left(D_{\left(p_{i}, p_{i+1}\right)}+D_{\left(p_{i+1}, p_{i}\right)}\right)\right],
$$

where the minimum is taken over all finite sequences $p_{1}, p_{2}, \ldots, p_{n} \in \Sigma^{\prime}(f)$ with $p_{1}=p, p_{n}=p^{\prime}$. It follows from (1.7) that $d($,$) satisfies the triangle inequality$ and is symmetric. It follows from 1.6(a)-(c) and (1.7) that $d($,$) satisfies the other$ properties of being a metric for $\Sigma^{\prime}(f)$. Finally 1.2(c), (d) follows from 1.6(c), (d) and (1.7).

Now property $1.2(\mathrm{e})$ will be verified. Recall that each factor $Y_{i}$ is homeomorphic to an open subset of $R^{k}$. So by 1.2(c), (d) it follows that each $W^{u}(x)$ is a one-to-one continuous image of $R^{k}$. Towards verifying $1.2(\mathrm{e})$ for $W^{s}(x)$, choose $p, p^{\prime} \in W^{s}(x)$. It follows from 1.2(c) that there will be no loss of generality in assuming there is a neighborhood $W_{p, 1}=X_{p, 1} \times Y_{p, 1}$ for $p$ so that $p, p^{\prime} \in X_{p, 1} \times y$ for some $y \in Y_{p, 1}$. Thus there are representatives $\left(x_{0}, x_{1}, x_{2}, \ldots\right),\left(x_{0}^{\prime}, x_{1}^{\prime}, x_{2}^{\prime}, \ldots\right) \in \Sigma(f)$ for $p, p^{\prime}$ such that $x_{0}, x_{0}^{\prime} \in U_{i}$ with $\pi_{i}\left(x_{0}\right)=\pi_{i}\left(x_{0}^{\prime}\right)$ for some $i$. Using [4, 1.2(a), (c)], finite sequences of points $\left(x_{0, j}, x_{1, j}, x_{2, j}, x_{3, j}, \ldots, x_{n_{j}, j}\right)$ in $K$ can be constructed by induction over $j=0,1,2,3, \ldots$ to satisfy the following:

1.8. (a) $x_{0, j}=x_{j}, x_{n_{j}, j}=x_{j}^{\prime}, n_{0}=1$.

(b) There is a subsequence $\left(x_{0, j+1}, x_{1, j}^{\prime}, x_{2, j}^{\prime}, \ldots, x_{n_{j-1, j}}^{\prime} x_{n_{j+1}, j+1}\right)$ of each $\left(x_{0, j+1}, x_{1, j+1}, \ldots, x_{n_{j+1}, j+1}\right)$ which is mapped by $f: K \rightarrow K$ one-to-one onto $\left(x_{0, j}, x_{1, j}, x_{2, j}, \ldots, x_{n_{j}, j}\right)$.

(c) For each pair $x_{i, j}, x_{i+1, j}$ there is a $U_{k}$ so that $x_{i, j}, x_{i+1, j} \in U_{k}$ and $\pi_{k}\left(x_{i, j}\right)=$ $\pi_{k}\left(x_{i+1, j}\right)$.

We will use the notation $\left(x_{i, j}^{\prime}\right)^{\prime}=x_{i, j}^{(2)},\left(x_{i, j}^{(2)}\right)^{\prime}=x_{i, j}^{(3)}$ etc., in reference to $1.8(\mathrm{~b})$.

Let $p_{i j} \in \Sigma^{\prime}(f)$ denote the point represented by $\left(f^{j}\left(x_{i, j}\right), \ldots, f^{2}\left(x_{i, j}\right), f\left(x_{i, j}\right)\right.$, $\left.x_{i, j}, x_{i, j}^{\prime}, x_{i, j}^{(2)}, x_{i, j}^{(3)}, \ldots\right)$ in $\Sigma(f)$. It follows from $[4,1.2(\mathrm{~b})], 1.8(\mathrm{c}), 1.5-(1.7)$, that $\lim _{j \rightarrow \infty} d\left(p_{i, j}, p_{i+1, j}\right)=0$ uniformly in $i$. It follows from 1.8(a), (b) that $p_{0, j}=$ $p, p_{n_{j}, j}=p^{\prime}$ and each sequence $\left(p_{0, j}, p_{1, j}, \ldots, p_{n_{j}, j}\right)$ is a refinement of $\left(p_{0, j-1}\right.$, $\left.p_{1, j-1}, \ldots, p_{n_{j-1}, j-1}\right)$. Thus as $j \rightarrow \infty$ the sequence $\left(p_{0, j}, p_{1, j}, \ldots, p_{n_{j}, j}\right)$ converges to the points on a continuous path which connects $p$ to $p^{\prime}$ in $W^{s}(x)$.

It remains to verify $1.2(\mathrm{f})$. The following lemma will be needed. It will be proven at the end of this section.

A Markov partition for $F^{\prime}: \Sigma^{\prime}(f) \rightarrow \Sigma^{\prime}(f)$ consists of a finite set of compact subsets $\left\{P_{i}\right\}$ of $\Sigma^{\prime}(f)$ which satisfy the following properties. $i^{\prime}$.

1.9. (a) There are subsets $P_{i}^{s} \subset X_{i^{\prime}}, p_{i}^{u} \subset Y_{i^{\prime}}$, so that $P_{i}=P_{i}^{s} \times P_{i}^{u}$, for some 
(b) $\operatorname{Closure}\left(P_{i}^{0}\right)=P_{i}$, where $P_{i}^{0}$ is the interior of $P_{i}$. Moreover $P_{i}^{0} \cap P_{j}^{0}=\varnothing$ if $i \neq j$, and $\bigcup_{i} P_{i}=\Sigma^{\prime}(f)$.

(c) If $F^{\prime}\left(q \times\left(P_{i}^{u}\right)^{0}\right) \cap p \times P_{j}^{u} \neq \varnothing$, then $F^{\prime}\left(q \times P_{i}^{u}\right) \supset p \times P_{j}^{u}$ for any $q \in P_{i}^{s}, p \in$ $\left(P_{j}^{s}\right)^{0}$, any $i, j$. If $\left.F^{\prime-1}\left(\left(P_{i}^{s}\right)^{0} \times q\right)\right) \cap P_{j}^{s} \times p \neq \varnothing$, then $F^{\prime-1}\left(P_{i}^{s} \times q\right) \supset P_{j}^{s} \times p$ for any $q \in P_{i}^{u}, p \in\left(P_{j}^{u}\right)^{0}$, and any $i, j$.

LEMMA 1.10. Given $\delta>0$ there is a Markov partition $\left\{P_{i}\right\}$ for $F^{\prime}: \Sigma^{\prime}(f) \rightarrow$ $\Sigma^{\prime}(f)$ such that diameter $\left(P_{i}\right)<\delta$ for all $i$.

To complete the proof of $1.2(\mathrm{f})$ it will suffice to show that there is a sequence of finite open covers of $\Sigma^{\prime}(f),\left\{U_{i j}: i \in I_{j}\right\}, j=1,2,3, \ldots$, and a positive integer $N$ satisfying:

1.11. (a) The diameter of each open set in $\left\{U_{i j}: i \in I_{j}\right\}$ is less than $j^{-1}$.

(b) For each $j$ and any $U_{x, j}$, the number of open sets in $\left\{U_{i j}: j \in I_{j}\right\}$ which intersect $U_{x, j}$ is less than $N$.

Let $\left\{P_{k}\right\}$ denote a Markov partition for $F^{\prime}: \Sigma^{\prime}(f) \rightarrow \Sigma^{\prime}(f)$. A sequence of partitions $\left\{P_{k, j}\right\}$ can be constructed from $\left\{P_{k}\right\}$ as follows. $\left\{P_{k, 1}\right\}$ consist of all intersections $F^{\prime}\left(P_{x}\right) \cap F^{-1}\left(P_{y}\right)$, where $P_{x}, P_{y} \in\left\{P_{k}\right\}$ and $F^{\prime}\left(P_{x}^{0}\right) \cap F^{\prime-1}\left(P_{y}^{0}\right) \neq$ $\varnothing$. Assuming $\left\{P_{k, j}\right\}, 1 \leq j \leq r$, have been defined, let $\left\{P_{k, r+1}\right\}$ consists of all intersections $F^{\prime}\left(P_{x, r}\right) \cap F^{\prime-1}\left(P_{y, r}\right)$ such that $P_{x, r}, P_{y, r} \in\left\{P_{k, r}\right\}$ and $F^{\prime}\left(P_{x, r}^{0}\right) \cap$ $F^{\prime-1}\left(P_{y, r}^{0}\right) \neq \varnothing$.

It follows from $1.9,1.2(\mathrm{c}),(\mathrm{d})$, that if the diameters of all the sets $\left\{P_{k}\right\}$ are sufficiently small, then the following will hold.

1.12. (a) Each $\left\{P_{k, j}\right\}$ is a Markov partition for $F^{\prime}: \Sigma^{\prime}(f) \rightarrow \Sigma^{\prime}(f)$.

(b) There is a positive integer $N$, so that for each $j$ and each $P_{x, j}$ the number of sets in $\left\{P_{k, j}\right\}$ which intersect $P_{x, j}$ is less than $N$.

(c)

$$
\lim _{j \rightarrow \infty} \alpha_{j}=0, \quad \text { where } \alpha_{j} \equiv \max _{i \in I_{j}}\left(\operatorname{diameter}\left(P_{i j}\right)\right) .
$$

After choosing a subsequence of the $\left\{P_{k, j}: k \in I_{j}\right\}, j=1,2,3, \ldots$, we may assume (d) $\alpha_{j}<10^{-1} j^{-1}$.

Now choose open sets $\left\{U_{i j}: j \in I_{j}\right\}$ satisfying

1.13. (a) $U_{i j} \supset P_{i j}$,

(b) $U_{i j} \cap U_{i^{\prime} j} \neq \varnothing \Leftrightarrow P_{i j} \cap P_{i^{\prime} j} \neq \varnothing$.

Then $1.12,1.13$ imply that the $\left\{U_{i j}: i \in I_{j}\right\}, j=1,2,3, \ldots$, satisfy 1.11 .

This completes the proof of Proposition 1.2.

ProOF OF LEMMA 1.6. Properties 1.6(a), (b) are immediate from the definition of $D($,$) .$

To verify 1.6(d) note that $1.2(\mathrm{~d})$ (for $D($,$) in place of d($,$) ) follows from the$ expanding property for $f: K \rightarrow K$. Note also that 1.2(c) (for $D($,$) in place of$ $d()$,$) follows from [4,1.2(\mathrm{~b})]$ and 1.5 .

The following property will be needed for verifying $1.6(\mathrm{c})$.

1.14. There is a positive integer $m$, so that for any $x_{1}, x_{2}, x_{3} \in \Sigma^{\prime}(f)$, and any integer $l>m$ ( $m$ is independent of $\beta$ in 1.5), $x_{2} \in W_{x_{1}, l}, x_{3} \in W_{x_{2}, l} \Rightarrow x_{3} \in$ $W_{x_{1}, l-m}$.

Property 1.14 follows from $[4,1.2(\mathrm{~b}), 1.1]$ and 1.4 . The details of this argument are left to the reader. 
We will assume the contrary of $1.6(\mathrm{c})$, namely

1.15. (a) $\left(\left(\sum_{i=1}^{n-1} D\left(p_{i}, p_{i+1}\right)\right)<\gamma^{-1} D\left(p_{1}, p_{n}\right)\right.$, and derive a contradiction when

(b) $\gamma=10$,

(c) $1<\beta<\sqrt[m]{100 / 99}$ (where $m$ comes from 1.14).

Of all the different lengths $\left\{D\left(p_{i}, p_{i+1}\right): 1 \leq i \leq n-1\right\}$ let $\beta^{-l}, \beta^{-k}$ denote the minimum and maximum. Write $l=k+s m+r$, where $0 \leq r<m ; m$ comes from 1.14. For each integer $0 \leq j \leq s$ a subsequence $\left(p_{1, j}, p_{2, j}, \ldots, p_{n_{j}, j}\right)$ of $\left(p_{1}, p_{2}, \ldots, p_{n}\right)$ is constructed by the following inductive procedure. Set $p_{i, s} \equiv$ $p_{i}, n_{s}=n$. Assuming $\left(p_{1, j}, \ldots, p_{n_{j}, j}\right)$ has been constructed, for all $q \leq j \leq s$, consider all subsequences $\left(p_{a, q}, p_{a+1, q}, p_{a+2, q}, \ldots, p_{a+b, q}\right)$ of consecutive points in $\left(p_{1, q}, p_{2, q}, \ldots, p_{n_{q}, q}\right)$ which are maximal with respect to the property that $D\left(p_{a+x, q}, p_{a+x+1, q}\right) \leq \beta^{-m q-k}$. In each such maximal subsequence discard every other point (i.e. discard $p_{a+1, q}, p_{a+3, q}, p_{a+5, q}$, etc.) with the exception of $p_{a, q}$ and $p_{a+b, q}$. Having discarded all these points, the remaining points are the sequence $\left(p_{1, q-1}, p_{2, q-1}, \ldots, p_{n_{q-1}, q-1}\right)$. The following property is deduced from 1.14.

1.16. If two consecutive points $p_{c, q-1}, p_{c+1, q-1}$ of $\left(p_{1, q-1}, p_{2, q-1}, \ldots, p_{n_{q-1}, q-1}\right)$ are not consecutive points of $\left(p_{1, q}, p_{2, q}, \ldots, p_{n_{q}, q}\right)$, then $D\left(p_{c, q-1}, p_{c+1, q-1}\right) \leq$ $\beta^{-m(q-1)-k}$.

By applying 1.16 to each step of the above inductive construction one gets

1.17. $\left(p_{1,0}, p_{2,0}, \ldots, p_{n_{0}, 0}\right)$ is a sequence starting at $p_{1}=p_{1,0}$ and ending at $p_{n}=p_{n_{0}, 0}$, such that $D\left(p_{i, 0}, p_{i+1,0}\right) \leq \beta^{-k}$ for all $1 \leq i \leq n_{0}-1$.

A direct combinatorial argument (which will be given at the end of this section) based on 1.16 will give the following estimate of $n_{0}$ in 1.17 .

1.18. When 1.15(a), (c) are satisfied, we have $n_{0} \leq \max \left\{1,4 \gamma^{-1} \beta^{k} D\left(p_{1}, p_{n}\right)\right\}$.

At this point we continue with the inductive construction of the subsequences $\left(p_{1, q}, p_{2, q}, \ldots, p_{n_{q}, q}\right)$, extending the above construction for $0 \leq q \leq s$ to negative values of $q$. It is deduced from $1.14,1.17$ that

1.19. (a) for each $q \leq 0,\left(p_{1, q} p_{2, q}, \ldots, p_{n_{q}, q}\right)$ is a sequence starting at $p_{1}=p_{1, q}$, ending at $p_{n}=p_{n_{q}, q}$, such that $D\left(p_{1, q}, p_{i+1, q}\right) \leq \beta^{-k-q m}$ for all $1 \leq i \leq n_{q}$.

(b) $n_{q} \leq \frac{3}{4} n_{q+1}$ for each $q \leq-1$ such that $n_{q+1}>2$.

Now write $n_{0}=\left(\frac{4}{3}\right)^{\alpha}$. Let $[\alpha]$ denote the least integer greater than $\alpha$. From 1.19(b) it follows that $n_{-[\alpha]}=2$, so by 1.19 (a) we have

(1.20) $D\left(p_{1}, p_{2}\right)<\beta^{-k+m[\alpha]}$.

Set $D\left(p_{1} p_{n}\right)=\beta^{-a}$. Then it follows from 1.15(b), (c), 1.18 that $m[\alpha]-k<-a$. This last inequality contradicts (1.20). This is the desired contradiction that 1.15 leads to.

This completes the proof of Lemma 1.6.

Proof OF LEMMA 1.10. Properties 1.2(a)-(d) allow the application of construction $[\mathbf{1}, 3.12]$ to get a Markov partition for $F^{\prime}$.

This completes the proof of Lemma 1.10 .

PROOF OF 1.18. For each $0 \leq q \leq s$ (where $l=k+s m+r, 0 \leq r<m$ and $m$ comes from 1.14) let $A_{q}$ be the set of pairs $\left(p_{i, q}, p_{i+1, q}\right)$ in the sequence $\left(p_{1, q}, p_{2, q}, \ldots, p_{n_{q}, q}\right)$ of 1.16 such that $D\left(p_{i, q}, p_{i+1, q}\right) \leq \beta^{-k-q m}$. Let $B_{q}$ denote the set of pairs $\left(p_{i, q}, p_{i+1, q}\right)$ such that $D\left(p_{i, q}, p_{i+1, q}\right) \leq \beta^{-k-q m}$, but $D\left(p_{i-1, q}, p_{i, q}\right)>$ $\beta^{-k-q m}$ and $D\left(p_{i+1, q} p_{i+2, q}\right)>\beta^{-k-q m}$ whenever $p_{i-1, q}$ or $p_{i+2, q}$ exist. Let $C_{q}$ denote the set of pairs $\left(p_{i}, p_{i+1}\right)$ in the original sequence $\left(p_{1}, p_{2}, \ldots, p_{n}\right)$ of $1.6(\mathrm{c})$ 
such that

$$
\beta^{-k-q m-m+1} \leq D\left(p_{i}, p_{i+1}\right) \leq \beta^{-k-q m} .
$$

Notice (see 1.16) that as the construction above is applied to the $\left\{p_{i, q}\right\}$ to get the $\left\{p_{i, q-1}\right\}$, the pairs in $A_{q}-B_{q}$ coalesce to fewer than $\frac{2}{3}\left(\left|A_{q}\right|-\left|B_{q}\right|\right)$ pairs in $A_{q-1}$, whereas each pair in $B_{q}$ remains a pair in $A_{q-1}$. Notice also (see 1.16) that $\left|B_{q} \cap\left(A_{q-1}-B_{q-1}\right)\right| \leq 2\left|C_{q-1}\right|$. (In the above formulae $|S|$ denotes the cardinality of the set $S$.)

Now a computation based on the estimates of the last paragraph shows

$$
n_{0} \leq \operatorname{maximum}\left[3\left(\sum_{q=0}^{s}\left(\frac{2}{3}\right)^{q} C_{q}\right), 1\right] \text {. }
$$

From 1.15(a), (c), and the definition of the $C_{q}$, we deduce

$$
3\left(\sum_{q=0}^{s}\left(\frac{2}{3}\right)^{q} C_{q}\right) \leq 4 \beta^{k} \gamma^{-1} D\left(p_{1}, p_{n}\right) .
$$

Finally 1.18 follows from (1.21), (1.22).

2. Codimension one quotient solenoids. In this section quotient solenoids associated to expanding immersions on one-dimensional branched manifolds are studied from a global point of view. A great portion of this section has been derived from the work of J. Franks [2] and A. Manning [5, 6]. The main result of this section (Proposition 2.1) will be used in the proof of Theorem 0.1 in $\S 3$.

Over any quotient solenoid $\Sigma^{\prime}(f)$ lies the vector bundle $T^{u}\left(\Sigma^{\prime}(f)\right)$ which is tangent to the unstable leaves of $F^{\prime}: \Sigma^{\prime}(f) \rightarrow \Sigma^{\prime}(f)$. In fact the $s_{j i}: Y_{j i} \rightarrow Y_{i j}$ of 1.1(b), 1.2(b), can be chosen to be $C^{r}$ diffeomorphisms (under the differentiability hypothesis of 1.2), and $T^{u}\left(\Sigma^{\prime}(f)\right)$ is then obtained by gluing together linear bundles $\left(X_{i} \times Y_{i}\right) \times R^{k}$ along linear isomorphisms

$$
\left(r_{j i} \times s_{j i}\right) \times d s_{j i}:\left(X_{j i} \times Y_{j i}\right) \times R^{k} \rightarrow\left(X_{i j} \times Y_{i j}\right) \times R^{k},
$$

where $k=\operatorname{dim}(K)$. Note that $F^{\prime}: \Sigma^{\prime}(f) \rightarrow \Sigma^{\prime}(f)$ is a local $C^{r}$-diffeomorphism in the local factors $Y_{i}$, so $F^{\prime}$ induces a bundle isomorphism $d^{u} F^{\prime}: T^{u}\left(\Sigma^{\prime}(f)\right) \rightarrow$ $T^{u}\left(\Sigma^{\prime}(f)\right)$.

A quotient solenoid $\Sigma^{\prime}(f)$ is a codimension one quotient solenoid if $T^{u}\left(\Sigma^{\prime}(f)\right)$ is a line bundle (i.e., if $\operatorname{dim}(K)=1$ ).

Proposition 2.1. Let $F^{\prime}: \Sigma^{\prime}(f) \rightarrow \Sigma^{\prime}(f)$ be the shift map on a codimension one qutoient solenoid (associated to an L.C.S. for $f: K \rightarrow K$ ), such that $T^{u}\left(\Sigma^{\prime}(f)\right.$ ) is orientable and $F^{\prime}$ has no wandering points. Suppose $F^{\prime}: \Sigma^{\prime}(f) \rightarrow \Sigma^{\prime}(f)$ has a fixed point $p \in \Sigma^{\prime}(f)$, such that $W^{u}(p)$ is dense in $\Sigma^{\prime}(f)$; and $d^{u} F^{\prime}: T^{u}\left(\Sigma^{\prime}(f)\right) \rightarrow$ $T^{u}\left(\Sigma^{\prime}(f)\right)$ preserves some orientation for $T^{u}\left(\Sigma^{\prime}(f)\right)$. Then there is a covering space $\hat{\rho}: \hat{\Sigma}^{\prime}(f) \rightarrow \Sigma^{\prime}(f)$ satisfying:

(a) The local cartesian product structure for $\Sigma^{\prime}(f)$ of $1.1,1.2$ lifts to a global cartesian product structure $\hat{\Sigma}^{\prime}(f)=X \times Y$, where $Y$ is the real line and $X$ is path connected. Moreover for each $x \in X, y \in Y, \rho: x \times Y \rightarrow \Sigma^{\prime}(f), \rho: X \times y \rightarrow \Sigma(f)$ are one-to-one immersions onto an unstable leaf, stable leaf of $F^{\prime}: \Sigma^{\prime}(f) \rightarrow \Sigma^{\prime}(f)$.

(b) $F^{\prime}: \Sigma^{\prime}(f) \rightarrow \Sigma^{\prime}(f)$ is covered by a homeomorphism $\hat{F}^{\prime}: \hat{\Sigma}^{\prime}(f) \rightarrow \hat{\Sigma}^{\prime}(f)$ which has a fixed point. 
(c) There is a free discrete group action $\phi: \pi \times \hat{\Sigma}^{\prime}(f) \rightarrow \hat{\Sigma}^{\prime}(f)$ of an additive subgroup of the real numbers, $\pi \subset R$, such that the quotient map $\hat{\Sigma}^{\prime}(f) \rightarrow \Sigma^{\prime}(f) / \phi$ (to the orbit space of $\phi$ ) equals the covering $\rho: \hat{\Sigma}^{\prime}(f) \rightarrow \Sigma^{\prime}(f)$. Moreover the isomorphism $F_{*}^{\prime}: \pi \rightarrow \pi$ defined by $\phi\left(F_{*}^{\prime}(\alpha), \hat{F}^{\prime}(x)\right)=\hat{F}^{\prime}(\phi(\alpha, x))$ for $\alpha \in \pi, x \in \hat{\Sigma}^{\prime}(f)$, is equal to multiplication (in $R$ ) by a number $\lambda>1$.

(d) $\pi$ is isomorphic to $Z^{n}$ for some positive integer $n$.

(e) $\pi \otimes_{Z} R=V_{s} \oplus V_{u}$, where

$$
F_{*}^{\prime} \otimes 1_{R}\left(V_{s}\right)=V_{s}, \quad F_{*}^{\prime} \otimes 1_{R}\left(V_{u}\right)=V_{u}, \quad \operatorname{dim}\left(V_{u}\right)=1,
$$

and $F_{*}^{\prime} \otimes 1_{R}$ is contracting on $V_{s}$ and expanding on $V_{u}$.

The following lemma shall be needed in the proof of Proposition 2.1(a), (b), (c).

LEMMA 2.2. Let $\Sigma^{\prime}(f)$ be a codimension one quotient solenoid with no wandering points. For each unstable leaf $W^{u}(p)$ of $F^{\prime}: \Sigma^{\prime}(f) \rightarrow \Sigma^{\prime}(f)($ see 1.2(e)) there is a metric $d_{p}($,$) on the set W^{u}(p)$ which satisfies the following properties.

(a) There is $\lambda>1$, independent of $p \in \Sigma^{\prime}(f)$, so that

$$
d_{F^{\prime}(p)}\left(F^{\prime}(x), F^{\prime}(y)\right)=\lambda d_{p}(x, y)
$$

for all $p \in \Sigma^{\prime}(f), x, y \in W^{u}(p)$.

(b) Let $N_{q}(F)$ denote the number of fixed points of $\left(F^{\prime}\right)^{q}: \Sigma^{\prime}(f) \rightarrow \Sigma^{\prime}(f)$. Then $\lim _{q \rightarrow \infty} N_{q}\left(F^{\prime}\right) / \lambda^{q}=1$.

(c) Each pair $\left(W^{u}(p), d_{p}(),\right)$ is isometric to the the real number line equipped with the Euclidean metric.

(d) For any subset $X_{i} \times Y_{i} \subset \Sigma^{\prime}(f)$ as in 1.1, 1.2, and any $x_{1}, x_{2} \in X_{i}, y_{1}, y_{2} \in$ $Y_{i}$, we have $d_{p}(p, q)=d_{p^{\prime}}\left(p^{\prime}, q^{\prime}\right)$, where $p=\left(x_{1}, y_{1}\right), q=\left(x_{1}, y_{2}\right), p^{\prime}=\left(x_{2}, y_{1}\right), q^{\prime}$ $=\left(x_{2}, y_{2}\right)$.

PROOF OF 2.2. Since $F^{\prime}: \Sigma^{\prime}(f) \rightarrow \Sigma^{\prime}(f)$ has a Markov partition (1.10) and no wandering points, the argument of $[\mathbf{8}, \S 4]$ applies to give a Borel measure $\mu$ on $\Sigma^{\prime}(f)$, satisfying

2.3. (a) $\mu(S) \neq 0$ for every open set in $\Sigma^{\prime}(f)$.

(b) $\mu_{\mid X_{i} \times Y_{i}}=\mu_{i} \times v_{i}$ for each $X_{i} \times Y_{i}$ of 1.1, 1.2, where $\mu_{i}, v_{i}$ are Borel measures on $X_{i}, Y_{i}$. Moreover, $\mu_{i \mid X_{i, j}}=\mu_{j \mid X_{j, i}}$ and $v_{i \mid Y_{i, j}}=v_{j \mid Y_{j, i}}$ (see 1.1 for $X_{i j}, Y_{i j}$ ).

(c) There is $\lambda>1$ so that for any Borel set $A \times B \subset X_{i} \times Y_{i}$ if $F^{\prime}(A \times B)=$ $A^{\prime} \times B^{\prime} \subset X_{j} \times Y_{j}$, then $v_{j}\left(B^{\prime}\right)=\lambda v_{i}(B)$.

Note that each $W^{u}(p)$ is the image of a one-one immersion of $R$ in $\Sigma^{\prime}(f)$ (see $1.2(\mathrm{e}))$. For $x, y \in W^{u}(p)$ let $[x, y]$ denote the subinterval of $W^{u}(p)$ with $x, y$ for endpoints. Choose $x_{1}, x_{2}, \ldots, x_{l} \in[x, y]$ so that $x=x_{1}, y=x_{l},\left[x_{i} x_{i+1}\right] \cap$ $\left[x_{i+1}, x_{i+2}\right]=x_{i+1},[x, y]=\bigcup_{i=1}^{l-1}\left[x_{i}, x_{i+1}\right],\left[x_{i}, x_{i+1}\right] \subset X_{i^{\prime}} \times Y_{i^{\prime}}$ for some $i^{\prime}$. Set $d_{p}(x, y) \equiv \sum_{i=1}^{l-1} v_{i^{\prime}}\left(\left[x_{i}, x_{i+1}\right]\right)$. Then, by $2.3(\mathrm{a})-(\mathrm{c}), d_{p}($,$) is well defined and$ satisfies $2.2(\mathrm{a}),(\mathrm{c}),(\mathrm{d})$.

The arguments used in $[5$, p. $425 ; \mathbf{6}$, p. 218$]$ apply here to show that $\lambda$ satisfies 2.2 (b).

This completes the proof of 2.2 .

ProOF OF Proposition 2.1. Choose $p \in \Sigma^{\prime}(f)$ a fixed point of $F^{\prime}$. Set $X \equiv W^{s, *}(p), Y \equiv W^{u, *}(p)$ in 2.1. Here $W^{u, *}(p)$ denotes the set $W^{u}(p)$ equipped with the topology defined by $d_{p}($,$) of 2.2 . W^{s, *}(p)$ is $W^{s}(p)$ equipped with the 
following topology: $U \subset W^{s}(p)$ is an open set if and only if there is an integer $q>0$ and $X_{i} \times Y_{i}$ as in 1.1, 1.2 so that $\left(F^{\prime}\right)^{q}(U)$ is an open subset of $X_{i} \times y$ for some $y \in Y_{i}$. Set $\hat{\Sigma}^{\prime} \equiv X \times Y$.

A continuous map $\rho: \hat{\Sigma}(f) \rightarrow \Sigma(f)$ is defined as follows. Let $r: X \rightarrow \Sigma^{\prime}(f), s: Y$ $\rightarrow \Sigma^{\prime}(f)$ denote the inclusions. Let $\rho(x, y) \in \Sigma^{\prime}(f)$ be the unique point satisfying the following.

2.4. (a) $\rho(x, y) \in W^{u}(r(x))$.

(b) $d_{r(x)}(r(x), \rho(x, y))=d_{p}(p, s(y))$.

(c) Orient the arcs $[r(x), \rho(x, y)],[p, s(y)]$ from $r(x)$ to $p(x, y)$ and from $p$ to $s(y)$. These orientations either both agree or both disagree with the orientations for $T^{u}\left(\Sigma^{\prime}(f)\right)$.

It follows from 2.2, 2.4, and the fact that each $W^{s}(p)$ is path connected (see 1.2(e)) that $\rho: \hat{\Sigma}^{\prime}(f) \rightarrow \rho\left(\Sigma^{\prime}(f)\right)$ is a covering map onto a compact component of $\Sigma^{\prime}(f)$ satisfying $2.1(\mathrm{a})$. Note that $\rho\left(\hat{\Sigma}^{\prime}(f)\right) \supset W^{u}(p)$ and, by the hypothesis of 2.1, $W^{u}(p)$ is dense in $\Sigma^{\prime}(f)$. So $\rho\left(\hat{\Sigma}^{\prime}(f)\right)=\Sigma^{\prime}(f)$, and $\rho: \hat{\Sigma}^{\prime}(f) \rightarrow \Sigma^{\prime}(f)$ is a covering map satisfying $2.1(\mathrm{a})$.

Now 2.1(b) will be verified. Two homeomorphisms $\hat{F}_{s}^{\prime}: X \rightarrow X, \hat{F}_{u}^{\prime}: Y \rightarrow Y$ can be defined by $\hat{F}_{s}^{\prime} \equiv F_{\mid W^{s}(p)}^{\prime}, \hat{F}_{u}^{\prime} \equiv F_{\mid W^{u}(p)}$. Now set

$$
\hat{F}^{\prime} \equiv \hat{F}_{s}^{\prime} \times \hat{F}_{u}^{\prime} \text {. }
$$

It follows from $2.2(\mathrm{a}), 2.4,(2.5)$ that $\hat{F}^{\prime}: \hat{\Sigma}^{\prime}(f) \rightarrow \hat{\Sigma}^{\prime}(f)$ is a lifting of $F^{\prime}: \Sigma^{\prime}(f) \rightarrow$ $\Sigma^{\prime}(f)$. Note that $(p, p) \in X \times Y$ is a fixed point for $\hat{F}^{\prime}$.

Towards verifying 2.1(c), we first construct for any $a, b \in \hat{\Sigma}^{\prime}(f)$, with $\rho(a)=\rho(b)$, a covering transformation $h_{a, b}: \hat{\Sigma}^{\prime}(f) \rightarrow \hat{\Sigma}^{\prime}(f)$ such that $h_{a, b}(a)=b$. Write $a=$ $(x, y), b=\left(x^{\prime}, y^{\prime}\right)$ in $\hat{\Sigma}^{\prime}(f)=X \times Y$. Define mappings $h_{x, x^{\prime}}: X \rightarrow X, h_{y, y^{\prime}}: Y \rightarrow Y$ to be the compositions

2.6. (a)

$$
\underset{h_{y, y^{\prime}}}{X=X \times y \stackrel{\rho}{\rightarrow} W^{s}\left(\rho(a) \stackrel{\rho^{-1}}{\rightarrow} X \times y^{\prime}=X\right.}
$$

and

$$
Y=x \times Y \stackrel{\rho}{\rightarrow} W^{u}(\rho(a)) \stackrel{\rho^{-1}}{\rightarrow} x^{\prime} \times Y=Y .
$$

Now set

(b) $h_{a, b} \equiv h_{y, y^{\prime}} \times h_{x, x^{\prime}}$.

The following properties can be deduced from 2.4, (2.5), 2.6 and the fact that for all $a=(x, y)$ in $\hat{\Sigma}^{\prime}(f)$ each map $\rho: X \times y \rightarrow W^{s}(\rho(a)), \rho: x \times Y \rightarrow W^{u}(\rho(a))$ is a one-to-one immersion which is onto $(2.1(\mathrm{a}))$.

2.7. (a) $h_{a, b}$ is a well-defined homeomorphism.

(b) $h_{a, b}(a)=b$, and $\rho\left(h_{a, b}(c)\right)=\rho(c)$ for all $c \in \Sigma^{\prime}(f)$.

(c) If $a=b$ then $h_{a, b}=1$. If $a \neq b$ then both $h_{x, x^{\prime}}, h_{y, y^{\prime}}$ are fixed point free.

(d) If $\rho(a)=\rho(b)=\rho(c)$ then $h_{b c} \circ h_{a b}=h_{a, c}$.

(e) $h_{x, x^{\prime}}: Y \rightarrow Y$ is an isometry of $\left(W^{u, *}(p), d_{p}(),\right)$.

Now set $\pi \equiv\left\{h_{a, b}: \rho(a)=\rho(b), a, b \in \hat{\Sigma}(f)\right\}$. Properties 2.7(a), (c), (d) imply that $\pi$ is a subgroup of the group of homeomorphisms on $\hat{\Sigma}^{\prime}(f)$. Define $\phi: \pi \times$ 
$\hat{\Sigma}^{\prime}(f) \rightarrow \hat{\Sigma}^{\prime}(f)$ by $\phi\left(h_{a, b}, c\right) \equiv h_{a, b}(c)$. Then properties $2.7(\mathrm{~b}),(\mathrm{c})$ imply that $\phi$ is a free discrete group action such that the quotient map $\hat{\Sigma}^{\prime}(f) \rightarrow \hat{\Sigma}^{\prime}(f) / \phi$ equals $\rho: \hat{\Sigma}^{\prime}(f) \rightarrow \Sigma^{\prime}(f)$.

It follows from $2.2(\mathrm{c}), 2.7(\mathrm{e})$, that each $h_{x, x^{\prime}}: Y \rightarrow Y$ is a translation of the real numbers, or the composition of a translation with multiplication by -1 . The hypothesis (of 2.1) that $f: K \rightarrow K$ preserve the orientation of $K$ rules out the second of these two possibilities. Thus $h_{a, b} \rightarrow h_{y, y^{\prime}}$ defines a homomorphism $g: \pi \rightarrow$ $R$ (by identifying translations with real numbers). It follows from 2.7(c) that $g$ is one-to-one, showing that $\pi$ is an additive subgroup of $R$.

A mapping $F_{*}^{\prime}: \pi \rightarrow \pi$ is defined by

$$
F_{*}^{\prime}\left(h_{a, b}\right) \equiv h_{\hat{F}^{\prime}(a), \hat{F}^{\prime}(b)} .
$$

It follows from $2.7(\mathrm{c}),(\mathrm{d})$ that $F_{*}^{\prime}$ is a homomorphism. Note that $F_{*}^{\prime}$ is an isomorphism, in fact $\left(F_{*}^{\prime}\right)^{-1}\left(h_{a, b}\right)=h_{\hat{F}^{\prime-1}(a), \hat{F}^{\prime-1}(b)}$. The equality $\phi\left(F_{*}^{\prime}(\alpha), \hat{F}^{\prime}(x)\right)=$ $\hat{F}^{\prime}(\phi(\alpha, x))$ of $2.1(\mathrm{c})$ is equivalent (by $\left.(2.8)\right)$ to $h_{a, b}=\hat{F}^{\prime-1} \circ h_{\hat{F}^{\prime}(a), \hat{F}^{\prime}(b)} \circ \hat{F}^{\prime}$, which is left for the reader to check. Finally it follows from 2.2(a), (2.8) that $\lambda g=g \circ F_{*}^{*}$, where $g: \pi \rightarrow R$ is the embedding of the previous paragraph and $\lambda>1$ comes from 2.2(a). This completes the proof of 2.1(c).

Now 2.1(d) will be verified. For each open set $W_{i}=X_{i} \times Y_{i}$ of 1.1, 1.2 and any $(r, s) \in X_{i} \times Y_{i}$, and any $(\hat{r}, \hat{s}) \in X \times Y$ with $\rho(\hat{r}, \hat{s})=(r, s)$, there are subsets $\hat{X}_{i} \subset X, \hat{Y}_{i} \subset Y$ uniquely determined by the following properties (here we make the assumption that each $Y_{i}$ is a connected interval).

2.9. (a) $\hat{r} \in \hat{X}_{i}, \hat{s} \in \hat{Y}_{i}$.

(b) $\rho: \hat{X}_{i} \times \hat{Y}_{i} \rightarrow X_{i} \times Y_{i}$ is a homeomorphism.

For each positive integer $m$, let $\pi^{m} \subset \pi$ denote the subset of all $h_{a, b}$ (of 2.6(b)) such that there is a sequence $\hat{X}_{i_{j}} \times \hat{Y}_{i_{j}}, j=1,2, \ldots, m$, of sets as in 2.9, with $a \in \hat{X}_{i_{1}} \times \hat{Y}_{i_{1}}, b \in \hat{X}_{i_{m}} \times \hat{Y}_{i_{m}},\left(\hat{X}_{i_{j}} \times \hat{Y}_{i_{j}}\right) \cap\left(\hat{X}_{i_{j+1}} \times \hat{Y}_{i_{j+1}}\right) \neq \varnothing$ for $j=1,2, \ldots, m-1$. Each $\pi^{m}$ is a finite subset of $\pi$. To see this, note for any $h_{a, b} \in \pi^{m}, d_{p}\left(y, y^{\prime}\right)$ can only take on a finite number of values depending on $m$ (here $y, y^{\prime}$ are the $Y$ coordinates of $a, b)$. Since $h_{y, y^{\prime}}: Y \rightarrow Y$ is translation by $\pm d_{p}\left(y, y^{\prime}\right)$, it follows from 2.7 (c) that $\pi^{m}$ is a finite subset.

Note $\Sigma^{\prime}(f)$ is path connected (2.1(a)) and compact. From this it follows that for large enough $m, \pi^{m}$ will generate $\pi$. Since $\pi^{m}$ is a finite set and $\pi \subset R$ (by $2.1(\mathrm{c})$ ), it follows that $\pi=Z^{n}$ for some positive integer $n$.

This completes the proof of $2.1(\mathrm{~d})$.

Before proceeding with the proof of 2.1(e), two additional lemmas will be stated and proven.

LEMMA 2.10 (A. MANNING [5]). Let $h: T^{n} \rightarrow T^{n}$ denote an automorphism of the $n$-torus, covered by the linear isomorphism $\hat{h}: R^{n} \rightarrow R^{n}$. Let $\lambda_{1}, \lambda_{2}, \ldots, \lambda_{n}$ denote eigenvalues of $\hat{h}$ (counted with multiplicity). Suppose the following are satisfied.

(a) $\left|\lambda_{1}\right|>1$.

(b) No $\lambda_{i}$ is a root of unity.

(c) $\lim _{m \rightarrow \infty}\left(\left|L\left(h^{m}\right)\right| /\left|\lambda_{1}^{m}\right|\right)=1$, where $L\left(h^{m}\right)$ is the Lefschetz number of $h^{m}$.

Then $\left|\lambda_{i}\right|<1$ for all $1<i \leq n$. 
ProOF OF 2.10. Since $L\left(h^{m}\right)=\prod_{i=1}^{n}\left(1-\lambda_{i}^{m}\right)$ (see $[\mathbf{9}$, p. 769]), we have $\log \left(\left|L\left(h^{m}\right)\right|\right)=\sum_{i=1}^{n} \log \left(\left|1-\lambda_{i}^{m}\right|\right)$. So

$$
\lim _{m \rightarrow \infty} \frac{\log \left(\left|L\left(h^{m}\right)\right|\right)}{m}=\sum_{\left|\lambda_{i}\right|>1} \log \left(\left|\lambda_{i}\right|\right)+\lim _{m \rightarrow \infty}\left(\frac{\sum_{\left|\lambda_{i}\right|=1} \log \left(\left|1-\lambda_{i}^{m}\right|\right)}{m}\right) .
$$

By $2.10(\mathrm{c})$ the

$$
P_{m} \equiv \frac{\sum_{\left|\lambda_{i}\right|=1} \log \left(\left|1-\lambda_{i}^{m}\right|\right)}{m}
$$

do converge to some limit $L$. Moreover, by $2.10(\mathrm{~b})$, the argument from [5, p. 425] applies to show $L=0$. So we have

$$
\lim _{m \rightarrow \infty} \frac{\log \left(\left|L\left(h^{m}\right)\right|\right)}{m}=\sum_{\left|\lambda_{i}\right|>1} \log \left(\left|\lambda_{i}\right|\right) .
$$

This last equality together with $2.10(\mathrm{a})$, (c) yields $\left|\lambda_{i}\right| \leq 1$ for $2 \leq i \leq n$.

To see that $\left|\lambda_{i}\right|<1$ for $2 \leq i \leq n$ note that

$$
\frac{L\left(h^{m}\right)}{\left|\lambda_{1}^{m}\right|}=\left|1-\lambda_{1}^{-m}\right|\left(\prod_{\left|\lambda_{i}\right|<1}\left|1-\lambda_{i}^{m}\right|\right)\left(\prod_{\left|\lambda_{i}\right|=1}\left|1-\lambda_{i}^{m}\right|\right) .
$$

The left-hand side of 2.11 tends to 1 (see 2.10(c)), so it follows (from 2.11) that

$$
\lim _{m \rightarrow \infty} \prod_{\left|\lambda_{i}\right|=1}\left|1-\lambda_{i}^{m}\right|=1 \text {. }
$$

This last equality leads to a contradiction because $\left|1-\lambda_{i}^{m}\right|<2$ and $\lambda_{i}^{m}$ gets arbitrarily close to 1 if $\left|\lambda_{i}\right|=1$.

This completes the proof of 2.10 .

LEMmA 2.12. Let $f: K \rightarrow K$, and $Z^{n}$ be as in 2.1(d). There is an integer $T>0$, such that for every integer $i \geq T$ there is a map $g_{i}: L_{i} \rightarrow L_{i}$ of a finite simplicial complex $L_{i}$, and a lifting $\hat{g}_{i}: \hat{L}_{i} \rightarrow \hat{L}_{i}$ of $g_{i}$ to a map of a covering space of $L_{i}$ so that the following are satisfied.

(a) $\hat{L}_{i} \rightarrow L_{i}$ is a regular covering of $L_{i}$ having $Z^{n}$ for the group of covering transformations. $\hat{L}_{i}$ is path connected.

(b) Let $g_{i *}: Z^{n} \rightarrow Z^{n}$ denote the homomorphism of $Z^{n}$ induced by $\hat{g}_{i}$ (i.e. $\hat{g}_{i} \circ \alpha=$ $g_{i *}(\alpha) \circ \hat{g}_{i}$ for $\left.\alpha \in Z^{n}\right)$. Then $g_{i *}=\left(F_{*}^{\prime}\right)^{i}$, where $F_{*}^{\prime}$ comes from $2.1(\mathrm{c})$.

(c) Let $N_{i}\left(F^{\prime}\right)$ denote the number of fixed points of $\left(F^{\prime}\right)^{i}: \Sigma^{\prime}(f) \rightarrow \Sigma^{\prime}(f)$. Then $L\left(g_{i}\right)=-N_{i}\left(F^{\prime}\right)$, where $L\left(g_{i}\right)$ is the Lefschetz number of $g_{i}$.

(d) For any $\beta \in \bar{H}_{*}\left(\hat{L}_{i}\right)$ there is a positive integer a so that $\bar{H}_{*}\left(\hat{g}_{i}^{a}\right)(\beta)=0$.

PROOF OF 2.12. There is an integer $T>0$ so that for all integers $i \geq T$ there is a Markov partition $\left\{P_{j}: j \in J\right\}$ (depending on $i$ ) for $\left(F^{\prime}\right)^{i}: \Sigma^{\prime}(f) \rightarrow \Sigma^{\prime}(f)$ which satisfies the following.

2.13. (a) Let $x_{1}, x_{2}, \ldots, x_{l} \in \Sigma^{\prime}(f)$ be the fixed points of $\left(F^{\prime}\right)^{i}: \Sigma^{\prime}(f) \rightarrow \Sigma^{\prime}(f)$. For each $x_{t}$ there is $P_{j_{t}}$ such that $x_{t} \in P_{j_{t}}^{0}$ and $P_{j_{t}}=P_{j_{t^{\prime}}} \Leftrightarrow x_{t}=x_{t^{\prime}}$.

(b) Each $P_{k}^{u}$ is a connected interval (see 1.9(a) for $P_{k}^{u}$ ).

(c) There is an integer $q>0$ (depending on $\left\{P_{k}\right\}$ ) satisfying the following. For any $X_{r} \times Y_{r}$ as in 1.1, and any $(x, y) \in X_{r} \times Y_{r}$, suppose $P_{j_{1}}, P_{j_{2}}, \ldots, P_{j_{m}}$ are 
a collection of the $\left\{P_{j}: j \in J\right\}$ so that for any one $P_{j_{t}}(1 \leq t \leq m)$ there is a $P_{j_{t^{\prime}}}\left(1 \leq t^{\prime} \leq m\right)$ such that $P_{j_{t^{\prime}}} \cap\left(x \times Y_{r}\right) \neq \varnothing$ and $P_{j_{t}} \cap P_{j_{t^{\prime}}} \neq \varnothing$. Then if for some other partition sets $P_{i_{1}}, P_{i_{2}}, \ldots, P_{i_{m}}$ it is true that $P_{i_{t}} \cap\left(F^{\prime}\right)^{i q}\left(P_{j_{t}}^{s} \times y\right) \neq \varnothing$ for all $1 \leq t \leq m$, then $\bigcap_{t=1}^{m} P_{i_{t}} \neq \varnothing$.

Towards verifying 2.13 , first note that $2.13(\mathrm{c})$ is satisfied by any Markov partition with the $P_{j}$ of sufficiently small diameters. As has been mentioned in the proof of 1.10 above, properties $1.2(\mathrm{a})-(\mathrm{d})$ allow the application of Sinai's construction (see $[8]$ ) to get a Markov partition for $\left(F^{\prime}\right)^{i}$ (if $i$ is sufficiently large). It is left to the reader to discover the very minor alterations of Sinai's construction needed to achieve 2.13(a), (b).

Define the simplicial complex $L_{i}$ to equal the simplicial nerve of the covering $\left\{P_{j}: j \in J\right\}$ of 2.13. By lifting the partition sets $P_{j}$ to $\hat{\Sigma}^{\prime}(f)$ (as in 2.9) we get a Markov partition $\left\{P_{\hat{j}}: \hat{j} \in \hat{J}\right\}$ for $\hat{F}^{\prime}: \hat{\Sigma}^{\prime}(f) \rightarrow \hat{\Sigma}^{\prime}(f)$. Let $\hat{L}_{i}$ equal the simplicial nerve of the covering $\left\{P_{\hat{j}}: \hat{j} \in \hat{J}\right\}$. Clearly $\hat{L}_{i} \rightarrow L_{i}$ is a regular covering having $Z^{n}$ for the group of covering transformations. Since $\hat{\Sigma}^{\prime}(f)$ is path connected $(2.1(\mathrm{a}))$, so is $\hat{L}_{i}$.

Towards defining $g_{i}: L_{i} \rightarrow L_{i}$, for each $k \in J$ set

$$
C_{k, i} \equiv\left\{\left(P_{j}:\left(F^{\prime}\right)^{i}\left(P_{k}^{0}\right) \cap P_{j}^{0} \neq \varnothing\right\} .\right.
$$

Each simplex $\Delta^{l} \in L_{i}$ corresponds to a nonempty intersection $\bigcap_{j \in I} P_{j}$ where $I \subset$ $J,|I|=l+1$ : set

$$
C_{i}\left(\Delta^{l}\right) \equiv \bigcup_{k \in I} C_{k, i}
$$

Let $\bar{C}_{i}\left(\Delta^{l}\right)$ denote the subcomplex of $L_{i}$ which is the simplicial nerve of the partition sets in $C_{i}\left(\Delta^{l}\right)$. Note

2.14. (a) $\bar{C}_{i}\left(\Delta^{k}\right) \subset \bar{C}_{i}\left(\Delta^{l}\right)$ if $\Delta^{k} \subset \Delta^{l}$.

A computation based on 2.13(b) and the definition of $\bar{C}_{i}\left(\Delta^{l}\right)$ will show that if the $\left\{P_{j}: j \in J\right\}$ have sufficiently small diameters then

(b) $\bar{C}_{i}\left(\Delta^{l}\right)$ is a collapsible simplicial complex.

Now use 2.14 to construct a map $g_{i}: L_{i} \rightarrow L_{i}$ satisfying

2.15. $g_{i}\left(\Delta^{l}\right) \subset \bar{C}_{i}\left(\Delta^{l}\right)$ for all $\Delta^{l} \in L_{i}$.

The $g_{i}: L_{i} \rightarrow L_{i}$ can be lifted to $\hat{g}_{i}: \hat{L}_{i} \rightarrow \hat{L}_{i}$ satisfying $2.12(\mathrm{~b})$.

Let $x_{t}, P_{j_{t}}$ be as in 2.13(a) and $P_{j_{t}^{\prime}}, P_{j_{t}^{\prime \prime}}$ are as pictured in 2.16 below. 2.16 .

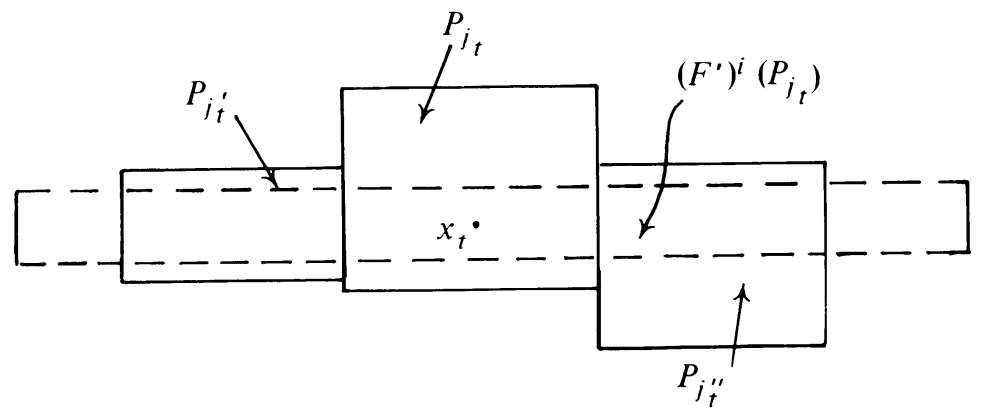


Let $V_{t}, V_{t}^{\prime}, V_{t}^{\prime \prime}$ be the vertices of $L_{i}$ corresponding to $P_{j_{t}}, P_{j_{t}^{\prime}}, P_{j_{t}^{\prime \prime}}$, and let $\left[V_{t}, V_{t}^{\prime}\right]$, $\left[V_{t}, V_{t}^{\prime \prime}\right]$ be the one simplices of $L_{i}$ corresponding to the nonempty intersections $P_{j_{t}} \cap P_{j_{t}^{\prime}}, P_{j_{t}} \cap P_{j_{t}^{\prime \prime}}$. Note, if the diameters of the $P_{j}$ (in 2.13) are sufficiently small, and $i$ is sufficiently large, then

2.17. $\bar{C}_{i}\left(\Delta^{k}\right) \cap\left(\Delta^{k}\right)^{0}=\varnothing$ if and only if $\Delta^{k} \neq V_{t},\left[V_{t}, V_{t^{\prime}}\right]$, or $\left[V_{t}, V_{t}^{\prime \prime}\right]$ for all $1 \leq t \leq l$.

Using 2.15-2.17, and the orientation hypothesis on $f: K \rightarrow K$, the $g_{i}: L_{i} \rightarrow L_{i}$ can be chosen so that

$$
\operatorname{trace}\left[H_{k}\left(g_{i}\right): H_{k}\left(L_{i}^{k}, L_{i}^{k-1}\right) \rightarrow H_{k}\left(L_{i}^{k}, L_{i}^{k-1}\right)\right]= \begin{cases}0, & \text { if } k \neq 0,1, \\ l, & \text { if } k=0 \\ 2 l, & \text { if } k=1\end{cases}
$$

where $L_{i}^{k}$ is the $k$ skeleton of $L_{i}$ and $l$ is the number of fixed points of $\left(F^{\prime}\right)^{i}: \Sigma^{\prime}(f) \rightarrow$ $\Sigma^{\prime}(f)$. So $L\left(g_{i}\right)=l-2 l=-N_{i}\left(F^{\prime}\right)$; which verifies $2.12(\mathrm{c})$.

It remains to verify $2.12(\mathrm{~d})$. Let $q$ be as in $2.13(\mathrm{c})$, and define for each $P_{\hat{k}}, \hat{k} \in \hat{J}$,

$$
C_{\hat{k}, q i} \equiv\left\{P_{\hat{j}}:\left(\hat{F}^{\prime}\right)^{i q}\left(P_{\hat{k}}^{0}\right) \cap P_{\hat{j}}^{0} \neq \varnothing\right\} .
$$

And define for each $\hat{\Delta}^{l} \in \hat{L}_{i}$,

$$
C_{q i}\left(\hat{\Delta}^{l}\right) \equiv \bigcup_{\hat{k} \in \hat{I}} C_{\hat{k}, q i}
$$

where $\hat{\Delta}^{l}$ corresponds to the nonempty intersection $\bigcap_{\hat{k} \in \hat{I}} P_{\hat{k}}$ with $\hat{I} \subset \hat{J}$ and $|\hat{I}|=$ $l+1$. Let $\bar{C}_{q i}\left(\hat{\Delta}^{l}\right)$ denote the simplicial nerve of $C_{q i}\left(\hat{\Delta}^{l}\right)$. Note

2.19. (a) $\bar{C}_{q i}\left(\hat{\Delta}^{k}\right) \subset \bar{C}_{q i}\left(\hat{\Delta}^{l}\right)$ if $\hat{\Delta}^{k} \subset \hat{\Delta}^{l}$, and from 2.13(b) it follows that

(b) $\hat{C}_{q i}\left(\hat{\Delta}^{l}\right)$ is a collapsible simplicial complex.

Using 2.19 , construct a continuous map $\hat{g}_{q i}: \hat{L}_{i} \rightarrow \hat{L}_{i}$ satisfying

2.20. (a) $\hat{g}_{q i}\left(\hat{\Delta}^{l}\right) \subset \bar{C}_{q i}\left(\hat{\Delta}^{l}\right)$.

It is left as an exercise to the reader (use 2.20(a) and 2.15) to show

(b) $\hat{g}_{q i}$ and $\left(\hat{g}_{i}\right)^{q}$ are homotopic.

By 2.20(b) it will suffice to verify 2.12 (d) for $\hat{g}_{q i}$ in place of $\hat{g}_{i}$. Let $(x, y) \in X \times Y$ denote the fixed point of $\hat{F}^{\prime}: \hat{\Sigma}^{\prime}(f) \rightarrow \hat{\Sigma}^{\prime}(f)($ see $2.1(\mathrm{~b}))$. Set

$$
D_{i} \equiv\left\{P_{\hat{j}}: P_{\hat{j}} \cap x \times Y \neq \varnothing\right\} .
$$

Set

$$
D_{i}^{\prime} \equiv\left\{P_{\hat{j}}: P_{\hat{j}} \cap P_{\hat{k}} \neq \varnothing \text { for some } P_{\hat{k}} \in D_{i}\right\} .
$$

Let $\bar{D}_{i}^{\prime}$ denote the simplicial nerve of the sets in $D_{i}^{\prime}$. To complete the verification of 2.12 (d) it will suffice to show

2.21. (a) $\hat{g}_{q i}\left(\bar{D}_{i}^{\prime}\right)$ is contained in a collapsible subcomplex $\bar{C}$ of $\bar{D}_{i}^{\prime}$.

(b) For each $\hat{\Delta}^{l} \in \hat{L}_{i}$ there is $b>0$ so that $\left(\hat{g}_{q i}\right)^{b}\left(\hat{\Delta}^{l}\right) \subset \bar{D}_{i}^{\prime}$.

To see $2.21(\mathrm{~b})$, note by construction of $\hat{F}^{\prime}: \hat{\Sigma}^{\prime}(f) \rightarrow \hat{\Sigma}^{\prime}(f)$, the fact that $(x, y)$ is the fixed point of $\hat{F}^{\prime}$, and by $1.2(\mathrm{c})-(\mathrm{e})$, all of $\hat{\Sigma}^{\prime}(f)$ converges to $x \times Y$ under repeated applications of $\hat{F}^{\prime}$. 
For this same reason $\hat{g}_{q i}\left(\bar{D}_{i}^{\prime}\right) \subset \bar{D}_{i}^{\prime}$. To get $\bar{C}$ of $2.21(\mathrm{a})$ set

$$
C_{q i}^{\prime}\left(P_{\hat{j}}\right) \equiv\left\{P_{\hat{k}}:\left(\hat{F}^{\prime}\right)^{i q}\left(P_{\hat{j}}\right) \cap P_{\hat{k}} \neq \varnothing\right\}, \quad C \equiv \bigcup_{P_{\hat{j}} \in D_{i}^{\prime}} C_{q i}^{\prime}\left(P_{\hat{j}}\right) .
$$

Let $\bar{C}$ denote the simplicial nerve of $C$. From 2.13(b), (c), it follows that $\bar{C}$ is collapsible, and $\bar{C} \subset \bar{D}_{i}^{\prime}$. Since $C_{q i}^{\prime}\left(P_{\hat{j}}\right) \supset C_{q i}\left(P_{\hat{j}}\right)$ for all $P_{\hat{j}}$, it follows that $\hat{g}_{q i}\left(\bar{D}_{i}^{\prime}\right) \subset \bar{C}$.

This completes the proof of 2.12 .

We now return to the

PROOF OF 2.1(e). Here is the plan for verifying 2.1(e). Let $\hat{h}: R^{n} \rightarrow R^{n}$ denote $\left(F_{*}^{\prime}\right) \otimes 1_{R}: Z^{n} \otimes_{Z} R \rightarrow Z^{n} \otimes R$. Since this $\hat{h}$ is an isomorphism on the integer lattice $Z^{n} \otimes_{Z} Z \subset Z^{n} \otimes_{Z} R$ (see 2.1(c)), it covers an automorphism $h: T^{n} \rightarrow T^{n}$ of the $n$-dimensional torus. We will see in the following paragraphs that $h, \hat{h}$ satisfy $2.10(\mathrm{a}),(\mathrm{b}),(\mathrm{c})$. Then the conclusion of 2.10 will imply $2.1(\mathrm{e})$.

Set $\lambda_{1}=\lambda$, where $\lambda$ comes from 2.1(c). Then 2.1(c) implies that 2.10(a) is satisfied by $h, \hat{h}$.

Now 2.10(b) will be verified. If $\lambda_{i}^{m}=1$, for some integer $m \geq 1$ and some eigenvalue $\lambda_{i}$ of $\hat{h}$, then there must be $\alpha \in Z^{n}, \alpha \neq 0$, so that $\left(F_{*}^{\prime}\right)^{m}(\alpha)=\alpha$. This contradicts $2.1(\mathrm{c})$.

Now 2.10 (c) will be verified. Let $g_{i}: L_{i} \rightarrow L_{i}, \hat{g}_{i}: \hat{L}_{i} \rightarrow \hat{L}_{i}, Z^{n} \times \hat{L}_{i} \rightarrow \hat{L}_{\bar{i}}$ be as in 2.12. It follows from 2.2(b), 2.12(c), and $\lambda=\lambda_{1}$, that $\lim _{m \rightarrow \infty}\left|L\left(g_{m}\right)\right| / \lambda_{1}^{m}=1$. So to verify 2.10 (c) it will suffice to show

2.22. $L\left(g_{i}\right)=L\left(h^{i}\right)$ for all integers $i>0$.

Towards verifying 2.22 let $\hat{Z}^{n} \times\left(\hat{L}_{i} \times R^{n}\right) \rightarrow \hat{L}_{i} \times R^{n}$ denote the diagonal action of $Z^{n} \times \hat{L}_{i} \rightarrow \hat{L}_{i}$ from $2.12\left(\right.$ a), and the covering transformation group $Z^{n} \times R^{n} \rightarrow R^{n}$ for $T^{n}$. Set $S_{i} \equiv \hat{L}_{i} \times R^{n} / Z^{n}$. The projections $\hat{L}_{i} \times R^{n} \rightarrow \hat{L}_{i}$, and $\hat{L}_{i} \times R^{n} \rightarrow R^{n}$, yield maps $u_{i}: S_{i} \rightarrow L_{i}, v_{i}: S_{i} \rightarrow T^{n}$. The map $\hat{g}_{i} \times \hat{h}^{i}: \hat{L}_{i} \times R^{n} \rightarrow \hat{L}_{i} \times R^{n}$ covers a map (see 2.12(b)) $G_{i}: S_{i} \rightarrow S_{i}$. The following are satisfied.

2.23. (a) $u_{i}: S_{i} \rightarrow L_{i}, v_{i}: S_{i} \rightarrow T^{n}$ are both fiber bundles with fibers $R^{n}, \hat{L}_{i}$ respectively.

(b) $h \circ v_{i}=v_{i} \circ G_{i}$ and $g_{i} \circ u_{i}=u_{i} \circ G_{i}$.

(c) $G_{i \mid v_{i}^{-1}(0)}: v_{i}^{-1}(0) \rightarrow v_{i}^{-1}(0)$ is equal to $\hat{g}_{i}: \hat{L}_{i} \rightarrow \hat{L}_{i}$.

(d) The action of $\pi_{1}\left(T^{n}\right)$ on $H_{*}\left(v_{i}^{-1}(0)\right)$ is the same as that induced on $H_{*}\left(\hat{L}_{i}\right)$ by $Z^{n} \times \hat{L}_{i} \rightarrow \hat{L}_{i}$ of $2.12($ a).

Let $\left\{E_{p, q}^{r}\right\}$ be the homology spectral sequence for the fibration $v_{i}: S_{i} \rightarrow T^{n}$. Let ${ }_{t} H_{*}\left(T^{n}, H_{q}\left(v_{i}^{-1}(0)\right)\right)$ denote the homology groups of $T^{n}$ with coefficients in the $Z\left(\pi_{1}\left(T^{n}\right)\right)$-module $H_{q}\left(v_{i}^{-1}(0)\right)$ (see $\left.2.23(\mathrm{~d})\right)$. Then

2.24. (a) $\left(E_{p, q}^{r}\right)$ converges to $H_{p+q}\left(S_{i}\right)$.

(b) $E_{p, q}^{2}={ }_{t} H_{p}\left(T^{n}, H_{q}\left(v_{i}^{-1}(0)\right)\right)$.

Let $H_{p, q}^{r}: E_{p, q}^{r} \rightarrow E_{p, q}^{r}$ be the homomorphism of the spectral sequence induced by $G_{i}($ see $2.23(\mathrm{~b}))$. Let

$$
J_{*, q}:{ }_{t} H_{*}\left(T^{n}, H_{q}\left(v_{i}^{-1}(0)\right)\right) \rightarrow{ }_{t} H\left(T^{n}, H_{q}\left(v_{i}^{-1}(0)\right)\right)
$$

be the homomorphism induced by $h^{i}: T^{n} \rightarrow T^{n}$ and $H_{q}\left(G_{i}\right): H_{q}\left(v_{i}^{-1}(0)\right) \rightarrow$ 
$H_{q}\left(v_{i}^{-1}(0)\right)$. Then

(c) $H_{p q}^{r}$ converges to $H_{p+q}\left(G_{i}\right)$.

(d) $H_{p, q}^{2}=J_{p, q}$.

(e) $J_{*, 0}$ is an isomorphism.

(f) For each $\beta \in{ }_{t} \bar{H}_{*}\left(T^{n}, H_{q}\left(v_{i}^{-1}(0)\right)\right)$ with $q>0$ there is $j>0$ so that $J_{*, q}^{j}(\beta)=$ 0 (see $2.12(\mathrm{~d})$ and $2.23(\mathrm{c})$ ).

It follows from $2.24(\mathrm{c})$-(f) that $E_{p, 0}^{2}$ terms all live to infinity in the spectral sequence, and if $\beta \in E_{p, q}^{2}, q>0$, lives to infinity then $\left(H_{*}\left(G_{i}\right)\right)^{m}(\beta)=0$ for some integer $m>0$. Moreover, $H_{*}\left(G_{i}\right)_{\mid E_{* 0}^{2}}=H_{*}\left(h^{i}\right)$ (see 2.24(a), (b), and 2.12(a)). Thus $L\left(G_{i}\right)=L\left(h^{i}\right)$. From $2.23(\mathrm{a})$, (b) we get $L\left(G_{i}\right)=L\left(g_{i}\right)$. Now 2.22 follows from the last two equalities.

3. Classification of codimension one quotient solenoids. In this section Theorem 0.1 is proven. Recall that Theorem 0.1 classifies only those shift maps on codimension one quotient solenoids which come from an L.C.S.

This section owes very much to the work of J. Franks [2].

PROOF OF THEOREM 0.1 . We shall first prove 0.1 under the additional hypothesis that

3.1. (a) $T^{u}\left(\Sigma^{\prime}(f)\right)$ has an orientation.

(b) $d^{u} F^{\prime}: T^{u}\left(\Sigma^{\prime}(f)\right) \rightarrow T^{u}\left(\Sigma^{\prime}(f)\right)$ is orientation preserving.

(c) $F^{\prime}: \Sigma^{\prime}(f) \rightarrow \Sigma^{\prime}(f)$ has no wandering points and has a fixed point $p \in \Sigma^{\prime}(f)$ such that $W^{u}(p)$ is dense in $\Sigma^{\prime}(f)$.

Note that 3.1 is exactly the hypothesis of Proposition 2.1. Let $\hat{h}: R^{n} \rightarrow R^{n}$ denote the isomorphism $F_{*}^{\prime} \otimes 1_{R}: \pi \otimes_{Z} R \rightarrow \pi \otimes_{Z} R$ of $2.1(\mathrm{e})$. Since $\hat{h}$ maps the integer lattice $Z^{n} \subset R^{n}$ onto itself, it covers an automorphism of the torus $h: T^{n} \rightarrow T^{n}$. By $2.1(\mathrm{e}), h: T^{n} \rightarrow T^{n}$ is a hyperbolic automorphism. The proof of 0.1 will be completed (for those $F^{\prime}: \Sigma^{\prime}(f) \rightarrow \Sigma^{\prime}(f)$ satisfying 3.1 ) by showing that $F^{\prime}: \Sigma^{\prime}(f) \rightarrow \Sigma^{\prime}(f)$ is topologically conjugate to $h: T^{n} \rightarrow T^{n}$. This is accomplished in the following steps.

Step 1. In this step a continuous map $k: \Sigma^{\prime}(f) \rightarrow T^{n}$ is constructed which satisfies the following.

3.2. (a) $k$ is covered by a continuous map $\hat{k}: \hat{\Sigma}^{\prime}(f) \rightarrow \hat{T}^{n}$, where $\hat{T}^{n}=R^{n}$ and $\hat{\Sigma}^{\prime}(f)$ is the covering space of 2.1 .

(b) Let $\phi: \pi \rightarrow \pi_{1}(T)$ denote the map satisfying $\hat{k}(\alpha(x))=\phi(\alpha)(\hat{k}(x))$ for all $x \in \hat{\Sigma}^{\prime}(f)$ and $\alpha \in \pi$. (Note that $\hat{\Sigma}^{\prime}(f)$ is path connected by $2.1(\mathrm{a}), 3.1$, so $\phi(\alpha)$ in the above equation is independent of $x$.) Then $\phi$ is an isomorphism satisfying the following.

(c) $\phi \circ F_{*}^{\prime}=h_{*} \circ \phi$, where $F_{*}^{\prime}: \pi \rightarrow \pi$ comes from $2.1(\mathrm{c})$ and $h_{*}: \pi_{1}\left(T^{n}\right) \rightarrow \pi_{1}\left(T^{n}\right)$ is induced by $h: T^{n} \rightarrow T^{n}$.

To get $k: \Sigma^{\prime}(f) \rightarrow T^{n}$ first choose an embedding $\hat{g}: \hat{\Sigma}^{\prime}(f) \rightarrow R^{L}(L=$ a large integer). Since $\hat{\Sigma}^{\prime}(f)$ is a separable metric space and of finite dimension (see 1.2(a), (f)), such a $\hat{g}$ exists $\left[3\right.$, Theorem V3]. Note $\pi=Z^{n}$ acts on $R^{L}$ by identifying the first $n$-coordinates of $R^{L}$ with $\pi \otimes_{Z} R$. Moreover the arguments used in the construction of $\hat{g}: \hat{\Sigma}^{\prime}(f) \rightarrow R^{L}$ in $[\mathbf{3}]$ can be adapted so as to choose $\hat{g}: \hat{\Sigma}^{\prime}(f) \rightarrow R^{L}$ to be a $\pi$-equivariant embedding. Let $g: \Sigma^{\prime}(f) \rightarrow R^{L} / \pi$ be the map which lifts to $\hat{g}$. Now choose a homotopy equivalence $r: R^{L} / \pi \rightarrow T^{n}$ and set $k: \Sigma^{\prime}(f) \rightarrow T^{n}$ equal to the composition $\Sigma^{\prime}(f) \stackrel{g}{\rightarrow} R^{L} / \pi \stackrel{r}{\rightarrow} T^{n}$. Then $k: \Sigma^{\prime}(f) \rightarrow T^{n}$ satisfies 
$3.2(\mathrm{a})$, (b). By construction of $h$ it follows that there is an $r: R^{L} / \pi \rightarrow T^{n}$ so that $k=r \circ g$ also satisfies $3.2(\mathrm{c})$.

Step 2 . In this step the map $k: \Sigma^{\prime}(f) \rightarrow T^{n}$ of 3.2 is modified so as to satisfy the following.

3.3. (a) $k \circ F^{\prime}=h \circ k$.

(b) $k: \Sigma^{\prime}(f) \rightarrow T^{n}$ still satisfies 3.2 .

The argument used to accomplish this modification is taken from the proof of $[\mathbf{2}, 4.2]$, which is reviewed now. In the notation of the proof of $[\mathbf{2}, 4.2]$, let

$$
\begin{aligned}
& N=\Sigma^{\prime}(f), \\
& \bar{N}=\hat{\Sigma}^{\prime}(f), \\
& \bar{g}: \bar{N} \rightarrow \bar{N} \text { equal } \hat{F}^{\prime}: \hat{\Sigma}^{\prime}(f) \rightarrow \hat{\Sigma}^{\prime}(f), \\
& M=T^{n}, \\
& \bar{M}=\hat{T}^{n}, \\
& \bar{f}: \bar{M} \rightarrow \bar{M} \text { equal } \hat{h}: \hat{T}^{n} \rightarrow \hat{T}^{n} .
\end{aligned}
$$

Set $H \equiv\{\beta: \bar{N} \rightarrow \bar{M} \mid \beta(\alpha(x))=\phi(\alpha)(\beta(x)), x \in \bar{N}, \alpha \in \pi\}$, where $\phi: \pi \rightarrow \pi_{1}(N)$ comes from 3.2(b). In [2, p. 80], it is argued that if $H \neq \varnothing$, then there is a unique $\beta \in H$ satisfying $\beta \circ \bar{g}=\bar{f} \circ \beta$. By $3.2, H \neq \varnothing$ because $\hat{k} \in H$. So let $k: \Sigma^{\prime}(f) \rightarrow T^{n}$ of 3.3 be the unique map which lifts to $\beta: \bar{N} \rightarrow \bar{M}$ satisfying $\beta \circ \bar{g}=\bar{f} \circ \beta$.

Step 3. In this step it is shown that $k: \Sigma^{\prime}(f) \rightarrow T^{n}$ of 3.3 is a homeomorphism. This will complete the proof of 0.1 for those $F^{\prime}: \Sigma^{\prime}(f) \rightarrow \Sigma^{\prime}(f)$ which satisfy 3.1.

It follows from 3.3 that $k: \Sigma^{\prime}(f) \rightarrow T^{n}$ maps stable and unstable leaves of $F^{\prime}$ into stable and unstable leaves of $h$. Then by 2.1 (a) it follows that $\hat{k}=\hat{k}_{s} \times \hat{k}_{u}$, where $\hat{k}_{s}: X \rightarrow V_{s}, \hat{k}_{u}: Y \rightarrow V_{u}$ and $\hat{\Sigma}^{\prime}(f)=X \times Y(2.1(\mathrm{a})), \hat{T}^{n}=V_{s} \times V_{u}(2.1(\mathrm{e}))$. Note that both the actions $\pi \times \hat{\Sigma}^{\prime}(f) \rightarrow \hat{\Sigma}^{\prime}(f), \pi_{1}\left(T^{n}\right) \times \hat{T}^{n} \rightarrow \hat{T}^{n}$ are products of actions $\pi \times X \rightarrow X, \pi \times Y \rightarrow Y$ and $\pi_{1}\left(T^{n}\right) \times V_{s} \rightarrow V_{s}, \pi_{1}\left(T^{n}\right) \times V_{u} \rightarrow V_{u}$, respectively. Now from 3.3, 3.2 we have $\hat{k}_{u}(\alpha(y))=\phi(\alpha) \hat{k}_{u}(y)$ for all $y \in Y, \alpha \in \pi$. Thus if $q \in \operatorname{Image}\left(\hat{k}_{u}\right)$, then the entire $\pi_{1}\left(T^{n}\right)$-orbit of $q$ will lie in Image $\left(\hat{k}_{u}\right)$. Each such orbit is dense in $V_{u}$, so Image $\left(\hat{k}_{u}\right)$ is dense in $V_{u}$. Since both $Y, V_{s}$ are copies of the real line $(2.1(\mathrm{a}),(\mathrm{e}))$ it follows that $\hat{k}_{u}: Y \rightarrow V_{u}$, is onto. Which implies that $k: \Sigma^{\prime}(f) \rightarrow T^{n}$ maps each unstable leaf of $\Sigma^{\prime}(f)$ onto an unstable leaf of $h: T^{n} \rightarrow T^{n}$. Since any unstable leaf of $h$ is dense in $T^{n}$, and $\Sigma^{\prime}(f)$ is compact, it follows that $k: \Sigma^{\prime}(f) \rightarrow T^{n}$ is onto.

It remains to show that $k: \Sigma^{\prime}(f) \rightarrow T^{n}$ is one-to-one. It will suffice to show that $\hat{k}: \hat{\Sigma}^{\prime}(f) \rightarrow \hat{T}^{n}$ is one-to-one. Choose a finite set of compact rectangles $A_{i} \times B_{i} \subset$ $V_{s} \times V_{u}, i=1,2, \ldots, a$, so that the sets $\left\{\gamma\left(A_{i} \times B_{i}\right): i=1,2, \ldots, a, \gamma \in \pi_{1}\left(T^{n}\right)\right\}$ form a covering for $\hat{T}^{n}$. Given $q \in T^{n}$ choose for each integer $j>0$ elements $\gamma_{j}, \gamma_{j}^{\prime} \in \pi_{1}\left(T^{n}\right)$ and integers $i_{j}, i_{j}^{\prime} \in\{1,2,3, \ldots, a\}$, so that

$$
q \in \underbrace{h^{j}\left(\gamma_{j}\left(A_{i_{j}} \times B_{i_{j}}\right)\right) \cap h^{-j}\left(\gamma_{j}^{\prime}\left(A_{i_{j}^{\prime}} \times B_{i_{j}^{\prime}}\right)\right)}_{U_{j}} .
$$

It will suffice to show that the diameter of $\hat{k}^{-1}\left(U_{j}\right)$ gets arbitrarily small as $j \rightarrow$ $\infty$. Towards this end note that it follows from $3.2-3.4,2.1(\mathrm{c})$, and the fact that 
$\hat{k}=\hat{k}_{s} \times \hat{k}_{u}$, that

$$
\begin{aligned}
\hat{k}^{-1}\left(U_{j}\right)= & {\left[F_{*}^{\prime j}\left(\phi^{-1}\left(\gamma_{j}\right)\right)\left(\hat{F}^{\prime j}\left(A_{i_{j}}^{\prime} \times B_{i_{j}}^{\prime}\right)\right)\right] } \\
& \cap\left[\hat{F}_{*}^{\prime-j}\left(\phi^{-1}\left(\gamma_{j}^{\prime}\right)\right)\left(\hat{F}^{\prime-j}\left(A_{i_{j}^{\prime}}^{\prime} \times B_{i_{j}^{\prime}}^{\prime}\right)\right)\right],
\end{aligned}
$$

where $A_{i_{j}}^{\prime}=\hat{k}_{s}^{-1}\left(A_{i_{j}}\right), A_{i_{j}^{\prime}}^{\prime}=\hat{k}_{s}^{-1}\left(A_{i_{j}^{\prime}}\right), B_{i_{j}}^{\prime}=\hat{k}_{u}^{-1}\left(B_{i_{j}}\right), B_{i_{j}^{\prime}}^{\prime}=\hat{k}_{u}^{-1}\left(B_{i_{j}^{\prime}}\right)$. Since $\hat{k}: \Sigma^{\prime}(f) \rightarrow \hat{T}^{n}$ is a covering of a map between compact spaces, each of the sets $A_{i_{j}}^{\prime}, A_{i_{j}^{\prime}}^{\prime}, B_{i_{j}}^{\prime}, B_{i_{j}^{\prime}}^{\prime}$ must be compact. So applying $1.2(\mathrm{c}),(\mathrm{d}),(\mathrm{e}), 2.1(\mathrm{a})$ we see that the diameters of $\hat{F}_{s}^{\prime j}\left(A_{i_{j}}^{\prime}\right), \hat{F}_{u}^{\prime-j}\left(B_{i_{j}^{\prime}}^{\prime}\right)$ get arbitrarily small as $j \rightarrow \infty$. It follows now from 3.5 that the diameter of $\hat{k}^{-1}\left(U_{j}\right)$ also gets arbitrarily small as $j \rightarrow \infty$.

This completes Step 3 and the proof of 0.1 if $F^{\prime}: \Sigma^{\prime}(f) \rightarrow \Sigma^{\prime}(f)$ satisfies 3.1.

Now 0.1 will be proven without the added hypothesis 3.1. First note that $\Sigma^{\prime}(f)$ has a finite number of compact path connected components (see 1.2(a), (e)) which will be denoted $\Sigma_{1}, \Sigma_{2}, \Sigma_{3}, \ldots, \Sigma_{l}$. Choose an integer $m>0$ so that $f^{\prime m}\left(\Sigma_{i}\right)=\Sigma_{i}$ for all $1 \leq i \leq l$. If $T^{u}\left(\Sigma_{i}\right)$ is not orientable there is a twofold cover $\rho_{i}: \stackrel{+}{\Sigma}_{i} \rightarrow \Sigma_{i}$ such that $\rho_{i}^{*}\left(T^{u}\left(\Sigma_{i}\right)\right)$ is orientable. The homeomorphism $F^{\prime m}: \Sigma_{i} \rightarrow \Sigma_{i}$ lifts to a homeomorphism $\stackrel{+}{F}_{i}: \stackrel{+}{\Sigma}_{i} \rightarrow \stackrel{+}{\Sigma}_{i}$ (the existence of the bundle isomorphism $d^{u} F^{\prime m}: T^{u}\left(\Sigma_{i}\right) \rightarrow T^{u}\left(\Sigma_{i}\right)$ guarantees the existence of the lifting $\left.\stackrel{+}{F}_{i}: \stackrel{+}{\Sigma}_{i} \rightarrow \stackrel{+}{\Sigma}_{i}\right)$.

The following lemma is proven at the end of this section.

LEMMA 3.6. For sufficiently large $m, F^{\prime m}: \Sigma_{i} \rightarrow \Sigma_{i}$ will satisfy 3.1 if $T^{u}\left(\Sigma_{i}\right)$ is orientable. If $T^{u}\left(\Sigma_{i}\right)$ is not orientable, then $\stackrel{+}{F}_{i^{\prime}}: \stackrel{+}{\Sigma}_{i} \rightarrow \stackrel{+}{\Sigma_{i}}$ will satisfy 3.1 . Moreover, each $F^{\prime m}: \Sigma_{i} \rightarrow \Sigma_{i}, \stackrel{+}{F}_{i}: \stackrel{+}{\Sigma}_{i} \rightarrow \stackrel{+}{\Sigma}_{i}$ is the shift map on a quotient solenoid associated to an L.C.S.

It follows from 3.6 and Steps 1-3 above, that either $F^{\prime m}: \Sigma_{i} \rightarrow \Sigma_{i}$ or $\stackrel{+}{F}_{i}: \stackrel{+}{\Sigma}_{i} \rightarrow$ $\stackrel{+}{\Sigma}_{i}$ is a hyperbolic automorphism on a torus $T^{n_{i}}$.

Note that in either case $\pi_{1}\left(\Sigma_{i}^{\prime}\right)=Z^{n_{i}}$. To see this let $\hat{\Sigma}_{i}$ be the universal cover of $\Sigma_{i}$ and $\hat{F}^{\prime m}: \hat{\Sigma}_{i} \rightarrow \hat{\Sigma}_{i}$ a lifting of $F^{\prime m}: \Sigma_{i} \rightarrow \Sigma_{i}$. Then $\hat{\Sigma}_{i}=X \times Y$, where $X \times y$ are the stable leaves of $\hat{F}^{\prime m}: \hat{\Sigma}_{i} \rightarrow \hat{\Sigma}_{i}$, and $x \times Y$ the unstable leaves. Pull back to $Y$ the metric on the unstable leaves of $F^{\prime m}: \Sigma_{i} \rightarrow \Sigma_{i}$ given in 2.2 , denoting the pullback by $d($,$) . Note (Y, d()$,$) is isometric to the real numbers with Euclidean$ distance $(2.2(\mathrm{c}))$, and the $Y$ factor of the covering transformations $\pi_{1}\left(\Sigma_{i}\right) \times \hat{\Sigma}_{i} \rightarrow \hat{\Sigma}_{i}$ gives a fixed point free action $\pi_{1}\left(\Sigma_{i}\right) \times Y \rightarrow Y$, which preserves the metric $d($,$) .$ It follows that $\pi_{1}\left(\Sigma_{i}\right)$ is a subgroup of the real numbers, so $\pi_{1}\left(\Sigma_{i}\right)=Z^{k}$ for some $k$. But $\pi_{1}\left(\stackrel{\Sigma}{\Sigma}_{i}\right)=Z^{n_{i}}$, so $k=n_{i}$.

Since $F^{\prime m}: \Sigma_{i} \rightarrow \Sigma_{i}$ or $\stackrel{+}{F}_{i}: \stackrel{+}{\Sigma}_{i} \rightarrow \stackrel{+}{\Sigma}_{i}$ is a hyperbolic automorphism of $T^{n_{i}}$, and $\pi_{1}\left(\Sigma_{i}\right)=Z^{n_{i}}$, it follows that $\pi_{1}\left(F^{\prime m}\right): \pi_{1}\left(\Sigma_{i}\right) \rightarrow \pi_{1}\left(\Sigma_{i}\right)$ is a hyperbolic isomorphism of $Z^{n_{i}}$. Define a homeomorphism $h: \bigcup_{i=1}^{l} T^{n_{i}} \rightarrow \bigcup_{i=1}^{l} T^{n_{i}}$ to be the disjoint union of group automorphisms $h_{i}: T^{n_{i}} \rightarrow T^{n_{i^{\prime}}}$, where $i^{\prime}$ is defined by $F^{\prime}\left(\Sigma_{i}\right)=\Sigma_{i^{\prime}}$, and $h_{i}$ is determined by stipulating that $\pi_{1}\left(h_{i}\right): \pi_{1}\left(T^{n_{i}}\right) \rightarrow \pi_{1}\left(T^{n_{i^{\prime}}}\right)$ 
must equal $\pi_{1}\left(F^{\prime}\right): \pi_{1}\left(\Sigma_{i}\right) \rightarrow \pi_{1}\left(\Sigma_{i^{\prime}}\right)$. Note that each $h^{m}: T^{n_{i}} \rightarrow T^{n_{i}}$ is a hyperbolic automorphism because $\pi_{1}\left(F^{\prime m}\right): \pi_{1}\left(\Sigma_{i}\right) \rightarrow \pi_{i}\left(\Sigma_{i}\right)$ is hyperbolic. It follows that $h: \bigcup_{i=1}^{l} T^{n_{i}} \rightarrow \bigcup_{i=1}^{l} T^{n_{i}}$ is an Anosov diffeomorphism. The proof of 0.1 is now completed by using the arguments of Steps 2 and 3 to show that $F^{\prime}: \Sigma^{\prime}(f) \rightarrow \Sigma^{\prime}(f)$ is topologically conjugate to $h: \bigcup_{i=1}^{l} T^{n_{i}} \rightarrow \bigcup_{i=1}^{l} T^{n_{i}}$.

This completes the proof of Theorem 0.1.

Proof OF Lemma 3.6. Assume $T^{u}\left(\Sigma_{i}\right)$ is oriented. For large enough $m$, $d^{u} F^{\prime m}: T^{u}\left(\Sigma_{i}\right) \rightarrow T^{u}\left(\Sigma_{i}\right)$ will preserve the orientation, and $F^{\prime m}$ will have a fixed point $p_{i} \in \Sigma_{i}$ (use 1.2 and 1.10 above). If we already knew that $F^{\prime m}: \Sigma_{i} \rightarrow \Sigma_{i}$ had no wandering points, then 1.2 , and the path connectedness of $\Sigma_{i}$, would allow the proof of $[2,1.8]$ to be applied to $F^{\prime m}: \Sigma_{i} \rightarrow \Sigma_{i}$ to show that $W^{u}\left(p_{i}\right)$ is dense in $\Sigma_{i}$. So to complete the verification of 3.1 for $F^{\prime m}: \Sigma_{i} \rightarrow \Sigma_{i}$ it remains to show the nonwandering property of 3.1(c). This is done in the following two steps.

Step 1. In this step it is shown that if $\bigcup_{q \in W^{u}(p)} W^{s}(q)=\Sigma_{i}$, for every $p \in \Sigma_{i}$, then $F^{\prime m}: \Sigma_{i} \rightarrow \Sigma_{i}$ has no wandering points.

Note if $F^{\prime m}: \Sigma_{i} \rightarrow \Sigma_{i}$ has wandering points then there is a Markov partition $\left\{P_{i}: i \in I\right\}$ for $F^{\prime m}: \Sigma_{i} \rightarrow \Sigma_{i}$ and a subset $J \subset I$ satisfying

3.7. (a) $J$ is a proper subset of $I$.

(b) $F^{\prime m}\left(P_{j}\right) \subset \bigcup_{i \in J} P_{i}$, if $j \in J$.

It follows from 3.7 that there is a fixed point $p \in \bigcup_{i \in J} P_{i}$ for $F^{\prime m}: \Sigma_{i} \rightarrow \Sigma_{i}$ so that $W^{u}(p) \subset \bigcup_{i \in J} P_{i}$. Since $\bigcup_{q \in W^{u}(p)} W^{s}(q)=\Sigma_{i}$, it follows that every point in $\Sigma_{i}$ is attracted towards $W^{u}(p)$, and hence towards $\bigcup_{i \in J} P_{i}$, under repeated applications of $F^{\prime m}: \Sigma_{i} \rightarrow \Sigma_{i}$. This leads to a contradiction, because $F^{\prime m}: \Sigma_{i} \rightarrow \Sigma_{i}$ must have periodic points in $\Sigma_{i}-\bigcup_{i \in J} P_{i}$.

Step 2 . In this step it is shown that $\bigcup_{q \in W^{u}(p)} W^{s}(q)=\Sigma_{i}$ for every $p \in \Sigma_{i}$.

It will suffice to show that there is a covering space $\rho: \hat{\Sigma}_{i} \rightarrow \Sigma_{i}$ satisfying 2.1(a). Towards this end the following claim will be useful. For $a, b \in W^{u}(a), a \in \Sigma_{i}$, let $[a, b] \subset W^{u}(a)$ denote the connected arc in $W^{u}(a)$ having $a, b$, as end points. For any path $g:[0,1] \rightarrow W^{s}(a)$, with $g(0)=a$, the translation of $[a, b]$ along the path $g$ is a family of arcs $[g(t), f(t)] \subset W^{u}(g(t))$ where $f:[0,1] \rightarrow W^{s}(b)$ is a path with $f(0)=b$.

Claim 3.8. (a) Given any $a \in \Sigma_{i}, b \in W^{u}(a), g:[0,1] \rightarrow W^{s}(b)$ with $g(0)=a$, there exists a translation of $[a, b]$ along $g$.

(b) Suppose $g, g^{\prime}:[0,1] \rightarrow W^{s}(a)$ are paths with $g(0)=g^{\prime}(0)=a, g(1)=g^{\prime}(1)$, and $[g(t), f(t)],\left[g^{\prime}(t), f^{\prime}(t)\right]$ denote the translation of $[a, b]$ along $g$ and $g^{\prime}$. Then $f(1)=f^{\prime}(1)$.

Before verifying 3.8, we use it to construct $\rho: \hat{\Sigma}_{i} \rightarrow \Sigma_{i}$. Choose any $p \in \Sigma_{i}$. Let $Y$ denote the point set $W^{u}(p)$ equipped with the topology which has the open intervals $\left\{[x, y]-(x \cup y): x, y \in W^{u}(p)\right\}$ for a base. Let $X$ denote the point set $W^{s}(p)$ equipped with the topology having the following sets for base: $U \subset X$ such that there is an integer $n>0$ and a product subset $X_{j} \times Y_{j} \subset \Sigma_{i}$ (as in 1.1) such that $f^{n}(U)=U^{\prime} \times q$ for $q \in Y_{j}, U^{\prime} \subset X_{j}$, where $U^{\prime}$ is an open subset of $X_{j}$. Set $\hat{\Sigma}_{i} \equiv X \times Y$. Define $\rho: \hat{\Sigma}_{i} \rightarrow \Sigma_{i}$ as follows. For $(x, y) \in X \times Y$ choose a path $g:[0,1] \rightarrow W^{s}(p)$ with $g(0)=p, g(1)=x$. Let $[g(t), f(t)]$ denote the translation of $[p, y]$ along $g$. Set $\rho(x, y) \equiv f(1)$. It follows from 3.8, and the path connectivity 
of $\Sigma_{i}$ and of each stable leaf in $\Sigma_{i}$, that $\rho: \hat{\Sigma}_{i} \rightarrow \Sigma_{i}$ is a covering map satisfying 2.1(a).

Now 3.8(a) will be verified. Fix a Riemann metric $\langle$,$\rangle on T^{u}\left(\Sigma_{i}\right)$, with which to measure the length of intervals $[a, b] \in W^{u}(a), a \in \Sigma_{i}$. The only way that 3.8 (a) could fail to be true would be that $[a, b]$ can be translated along $g:[0, \varepsilon) \rightarrow$ $W^{s}(a)$, for some $0<\varepsilon \leq 1$, and the length of $[g(t), f(t)]$ gets arbitrarily large as $t$ approaches $\varepsilon$ from the left (here $f:[0, \varepsilon) \rightarrow W^{s}(b)$ is an open ended path).

This would imply that for some $0<r, s<\varepsilon$ and subinterval $\left[a^{\prime}, b\right] \subset[a, b]$, and some $X_{j} \times Y_{j}$ of 1.1 , we have

3.9. (a) $\left[a_{s}^{\prime}, b_{s}^{\prime}\right],\left[a_{r}^{\prime}, b_{r}^{\prime}\right] \subset X_{j} \times Y_{j}$, where for each $t \in[0, \varepsilon)$ the $\left[g(t), a_{t}^{\prime}\right],\left[g(t), b_{t}^{\prime}\right]$ denote the translations of $\left[a, a^{\prime}\right],\left[a, b^{\prime}\right]$ along $g:[0, \varepsilon) \rightarrow W^{s}(a)$.

(b) The projection of $\left(a_{s}^{\prime}, b_{s}^{\prime}\right)$ to $Y_{j}$ contains the projection of $\left[a_{r}^{\prime}, b_{r}^{\prime}\right]$ to $Y_{j}$.

There is no loss of generality in assuming the $X_{j} \times Y_{j}$ of $1.1,1.2$ satisfy

(c) $\lim _{n \rightarrow \infty}\left(\operatorname{diameter}\left(F^{\prime n}\left(X_{j} \times q\right)\right)\right)=0$ for all $q \in Y_{j}$.

Now 3.9(a), (b) allow the choice of $c \in\left(a^{\prime}, b^{\prime}\right)$ and a sequence $c^{(i)} \in\left(a^{\prime}, b^{\prime}\right), i=$ $1,2,3, \ldots$, satisfying:

3.10. (a) $c_{r}, c_{s}$ project to the same point of $Y_{j}$.

(b) The $c_{r}^{(i)}, c_{s}^{(i)}$ project to different points of $Y_{j}$, for all $i$.

(c) $\lim _{i \rightarrow \infty} c^{(i)}=c$.

Note if all $c_{t}, r \leq t \leq s$, and $c_{t}^{(i)}, r \leq t \leq s$ and $i>0$, were in some $X_{k} \times$ $Y_{k}$ of $1.1,1.2$, then 3.10 (b) would lead to a contradiction (because translation within a $X_{k} \times Y_{k}$ must preserve the value of the $Y_{k}$-coordinate). In general, 1.2(c), 3.9 (c), 3.10(c), assure the existence of integers $m, n>0$, and an $X_{k} \times Y_{k}$, so that $F^{\prime n}\left(c_{t}\right), r \leq t \leq s, F^{\prime n}\left(c_{t}^{(i)}\right), r \leq t \leq s, i \geq m$, are all in $X_{k} \times Y_{k}$. Then by 3.10(b) $F^{\prime n}\left(c_{r}^{(i)}\right), F^{\prime n}\left(c_{s}^{(i)}\right)$, have different $Y_{k}$-coordinates, which is a contradiction as before. This completes the verification of $3.8(\mathrm{a})$.

Now $3.8(\mathrm{~b})$ will be verified. Suppose $f(1) \neq f^{\prime}(1)$. Then there is $c \in[a, b]$ and a sequence $c^{(i)} \in[a, b]$ satisfying

3.11. (a) All $c, c^{(i)}$ are contained in some one $X_{k} \times Y_{k}$ of 1.1 .

(b) $c_{1}=c_{1}^{\prime}$; here for any $x \in[a, b],\left[a, x_{t}\right]$ and $\left[a, x_{t}^{\prime}\right]$ denote the translations of $[a, x]$ along $g$ and $g^{\prime}$ of $3.8(\mathrm{~b})$.

(c) $c_{1}^{(i)}, c_{1}^{(i)^{\prime}}$ do not have the same $Y_{k}$-coordinate (for any $i$ ).

(d) $\lim _{i \rightarrow \infty} c^{(i)}=c$.

By $1.2(\mathrm{c}), 3.11(\mathrm{~b}),(\mathrm{d})$ there are integers $m, n>0$ and a $X_{j} \times Y_{j}$ of 1.1 , so that

$$
\bigcup_{t}\left[F^{\prime n}\left(c_{t}\right) \cup F^{\prime n}\left(c_{t}^{\prime}\right)\right] \subset X_{j} \times Y_{j}, \quad \bigcup_{t}\left[F^{\prime n}\left(c_{t}^{(i)}\right) \cup F^{\prime n}\left(c_{t}^{(i)^{\prime}}\right)\right] \subset X_{j} \times Y_{j}
$$

if $i \geq m$. By $3.11(\mathrm{c})$ the $F^{\prime n}\left(c_{1}^{(i)}\right), F^{\prime n}\left(c_{1}^{(i) \prime}\right)$ do not have the same $Y_{j}$-coordinates, which leads to a contradiction (because translation within $X_{j} \times Y_{j}$ must preserve the $Y_{k}$-coordinate values).

This completes the proof that $F^{\prime m}: \Sigma_{i} \rightarrow \Sigma_{i}$ satisfies 3.1. The proof that $\stackrel{+}{F}_{i}: \stackrel{+}{\Sigma}_{i} \rightarrow \stackrel{+}{\Sigma}_{i}$ satisfies 3.1 is carried out in a similar fashion.

It remains to show that $F^{\prime m}: \Sigma_{i} \rightarrow \Sigma_{i}, \stackrel{+}{F}_{i}: \stackrel{+}{\Sigma}_{i} \rightarrow \stackrel{+}{\Sigma}_{i}$ are shift maps on quotient solenoids associated to an L.C.S. To see this choose a Markov partition for $F^{\prime m}: \Sigma_{i} \rightarrow \Sigma_{i}$ (or $\stackrel{+}{F}_{i}: \stackrel{+}{\Sigma}_{i} \rightarrow \stackrel{+}{\Sigma}_{i}$ ) (1.10), and apply the constructions in the 
proofs of $[4,1.6,2.4]$ to the chosen Markov partition to realize $F^{\prime m}: \Sigma_{i} \rightarrow \Sigma_{i}$ (or $\left.\stackrel{+}{F}_{i}: \stackrel{+}{\Sigma}_{i} \rightarrow \stackrel{+}{\Sigma}_{i}\right)$ as the shift map on the quotient solenoid associated to W.L.C.S. This W.L.C.S. is actually an L.C.S. because the stable leaves of $F^{\prime m}: \Sigma_{i} \rightarrow \Sigma_{i}$ (or $\left.\stackrel{+}{F}_{i}: \stackrel{+}{\Sigma}_{i} \rightarrow \stackrel{+}{\Sigma}_{i}\right)$ are path connected $[\mathbf{4}, 1.6]$.

\section{REFERENCES}

1. R. Bowen, Equilibrium states and the ergodic theory of Anosov diffeomorphisms, Lecture Notes in Math., vol. 470, Springer, Berlin and New York, 1975.

2. J. Franks, Anosov diffeomorphisms, Proc. Sympos. Pure Math., vol. 14, Amer. Math. Soc., Providence, R. I., 1970, pp. 61-93.

3. W. Hurewicz and H. Wallman, Dimension theory, Princeton Univ. Press, Princeton, N. J., 1948.

4. L. Jones, Anosov diffeomorphisms and expanding immersions. I, preprint.

5. A. Manning, There are no new Anosov diffeomorphisms on tori, Amer. J. Math. 96 (1974), 422-429.

6. __ Axiom A diffeomorphisms have rational zeta functions, Bull. London Math. Soc. 3 (1971), 215-220.

7. S. E. Newhouse, On codimension one Anosov diffeomorphisms, Amer. J. Math. 92 (1970), 761-770.

8. Ya. Sinai, Markov partitions and C-diffeomorphisms, Functional Anal. Appl. 2 (1968), 61-82.

9. S. Smale, Differential dynamical systems, Bull. Amer. Math. Soc. 73 (1967), 747-817.

10. R. F. Williams, Expanding attractors, Inst. Hautes Études Sci. Publ. Math., No. 43, 1973, pp. 169-203.

Department of Mathematics, State University of NeW York, Stony Brook, NEW YORK 11794 